\title{
X-ray emission lines from inhomogeneous stellar winds
}

\author{
L. M. Oskinova, A. Feldmeier, and W.-R. Hamann
}

\author{
Astrophysik, Universität Potsdam, Am Neuen Palais 10, 14469 Potsdam, Germany \\ e-mail: lida@astro.physik.uni-potsdam.de
}

Received 2 February 2004 / Accepted 7 April 2004

\begin{abstract}
It is commonly adopted that X-rays from $\mathrm{O}$ stars are produced deep inside the stellar wind, and transported outwards through the bulk of the expanding matter which attenuates the radiation and affects the shape of emission line profiles. The ability of the X-ray observatories Chandra and XMM-Newton to resolve these lines spectroscopically provided a stringent test for the theory of the X-ray production. It turned out that none of the existing models was able to fit the observations consistently. The possible caveat of these models was the underlying assumption of a smooth stellar wind. Motivated by the evidence that the stellar winds are in fact structured, we present a 2-D numerical model of a stochastic, inhomogeneous wind. Small parcels of hot, X-ray emitting gas are permeated by cool, absorbing wind material which is compressed into thin shell fragments. Wind fragmentation alters the radiative transfer drastically, compared to homogeneous models of the same mass-loss rate. $\mathrm{X}$-rays produced deep inside the wind, which would be totally absorbed in a homogeneous flow, can effectively escape from a fragmented wind. The wind absorption becomes wavelength independent if the individual fragments are optically thick. The $\mathrm{X}$-ray line profiles are flat-topped in the blue part and decline steeply in the red part for the winds with a short acceleration zone. For the winds where the acceleration extends over significant distances, the lines can appear nearly symmetric and only slightly blueshifted, in contrast to the skewed, triangular line profiles typically obtained from homogeneous wind models of high optical depth. We show that profiles from a fragmented wind model can reproduce the observed line profiles from $\zeta$ Orionis. The present numerical modeling confirms the results from a previous study, where we derived analytical formulae from a statistical treatment.
\end{abstract}

Key words. stars: winds, outflows - X-rays: stars - line: profiles - radiative transfer

\section{Introduction}

Hot massive stars possess strong stellar winds, as discovered with the advent of UV spectroscopy (Morton 1967). One decade later it was detected that these stars emit X-rays (Harnden et al. 1979; Seward et al. 1979). The origin of these $\mathrm{X}$-rays is debated controversially. One of the first suggestions was the existence of a hot corona close to the stellar photosphere. However, the surrounding stellar wind should imprint strong K-shell absorption edges on the X-ray spectrum, which were not observed (Cassinelli \& Swank 1983). The basecoronal model was finally ruled out because of the lack of characteristic coronal emission lines (Baade \& Lucy 1982). Therefore it became commonly adopted that X-rays from hot stars are produced within their stellar winds, although most of the wind gas is obviously "cool".

The driving mechanism for the mass-loss from OB stars has been identified with radiation pressure on spectral lines. A corresponding theory was developed by Castor et al. (1975, "CAK"). After a number of improvements, this theory seems to correctly predict the observed mass-loss rates and wind velocities (Pauldrach et al. 1986). However, it was pointed out early (Lucy \& Solomon 1970), and later further investigated (Carlberg 1980; Owocki \& Rybicki 1984), that the stationary solution for a line-driven wind is unstable; small perturbations should grow quickly and form shocks. This "de-shadowing instability" is implicitly suppressed in the CAK theory.

The most detailed hydrodynamic modeling of this linedriven instability was presented by Feldmeier et al. (1997). These calculations show how initially small perturbations grow and form strong shocks that emit X-rays. This "wind shock model" was principally able to reproduce the X-ray flux from the $\mathrm{O}$ supergiant $\zeta$ Ori $\mathrm{A}$, which had been observed at that time only at low spectral resolution.

The ultimate test for models of X-ray production in stellar winds became possible with the launch of the X-ray observatories Chandra and XMM-Newton. Their spectrographs allow for the first time to resolve X-ray emission line profiles. Surprisingly, the shape of the X-ray line profiles turned out to be strikingly different among the few individual $\mathrm{O}$ stars observed so far:

$\theta^{1}$ OriC (Schulz et al. 2000) and $\zeta$ Ori A (Waldron \& Cassinelli 2001) show profiles which appear windbroadened, symmetric and unshifted with respect to the center wavelength;

$\delta$ Ori A (Miller et al. 2002) shows profiles which are narrower, symmetric, and also centered at the laboratory wavelength; 
$\zeta$ Pup (Cassinelli et al. 2001; Kahn et al. 2001) shows strongly broadened profiles which are blueshifted against the rest wavelength but otherwise appear to be symmetric.

If X-rays are produced deep inside the stellar wind, they have to propagate through the absorbing cool wind before they can emerge towards the observer. The opacity at X-rays energies is much larger than in the visual and UV, due to the K-shell absorption of abundant metal ions and the He II edge. Red-shifted photons of an emission line originate in the back hemisphere of the wind, and suffer much stronger absorption than photons in the blueshifted part of the line profile. Hence, as soon as the absorbing wind has a significant optical depth, the line profiles should be blueshifted and skewed, which means their shape resembles that of a triangle, with the flux maximum at maximum blueshift (MacFarlane et al. 1991; Ignace 2001). None of the observed stars meets this prediction.

Kramer et al. (2003) were able to fit eight X-ray lines observed in $\zeta$ Puppis with a homogeneous wind model. However, these fits could only be achieved by assuming much less absorption in the wind than expected from the generally adopted mass-loss rate of this star. Moreover, Kramer et al. (2003) state that there is not a strong trend of absorption with wavelength, in contrast to what is expected from the energy dependence of the mass absorption coefficient. Thus even for the prototype single $\mathrm{O}$ star $\zeta$ Puppis the wind shock model fails to reproduce observed X-ray emission lines consistently. At this point the question arose whether this model is adequate.

We emphasize that the whole interpretation of X-ray spectra had been based so far on over-simplified models, assuming a homogeneous distribution of the absorbing material. In contrast, there is strong empirical evidence that stellar winds are in fact strongly clumped (e.g., Hamann \& Koesterke 1998; Eversberg et al. 1998; Puls et al. 2003). This supports hydrodynamic models (Owocki et al. 1988; Feldmeier 1995) which show that most of the material in a radiatively driven wind is compressed into a series of dense shells by the instability. As the hydrodynamic models are one-dimensional, they cannot tell anything about the lateral structure of these wind inhomogeneities. The de-shadowing instability acts only in the radial direction; on the other hand, there is no obvious mechanism which could synchronize the wind in different directions. The observed variability in wind lines is not very pronounced, suggesting that the dense shells break up into a large number of fragments.

Wind inhomogeneity alters the radiative transfer significantly. We have studied the effects of wind fragmentation on the X-ray line formation in Feldmeier et al. (2003, Paper I), using a statistical approach that holds in the limit of infinitely many fragments. We showed analytically that the continuum wind opacity is greatly reduced in a structured wind, compared to a smooth wind with the same mass-loss rate. The line profiles we obtained are broad, blueshifted and flat-topped, i.e. symmetric, and thus of promising similarity to those observed in $\zeta$ Pup.

In the present paper we construct a stochastic wind model using a numerical approach. This allows to drop a number of idealizations in favor of a more realistic description.
Statistical approach. While Paper I relied on the statistical limit of very many fragments, we allow now for an arbitrary scale of fragmentation.

Wind geometry. We study models with different spatial distributions of fragments, both in the radial and lateral direction.

Wind velocity law. We abandon the restriction to a constant wind velocity and allow for so-called $\beta$-velocity laws.

Last not least, another purpose of the present paper is to better understand some surprising results obtained in Paper I. Despite of our focus on X-ray emission line formation, we want to emphasize their general importance for radiative transfer in inhomogeneous media.

In the next section we will introduce the stochastic wind model. Section 3 provides the formalism for solving the radiative transfer in a fragmented wind, as implemented in our numerical modeling. The basic effects of structured absorption on line profiles are illustrated in Sect. 4 using simplified examples. In Sect. 5 we introduce the concept of an effective opacity, and compare the line profiles obtained numerically from the stochastic wind model with the results from an analytical, statistical treatment. In Sect. 6 we evaluate line profiles using realistic assumptions and explore the parameter space. Conclusions are drawn in the final Sect. 7. A forthcoming paper will be devoted to detailed fits of observations.

\section{The model}

\subsection{Basic concepts}

Our model refers to the structure and physical conditions in the wind shock model according to the hydrodynamic simulations by Feldmeier et al. (1997). These dense flows driven by radiation pressure on spectral lines are highly supersonic. The de-shadowing instability leads to strong gas compression. Feldmeier et al. (1997) considered photospheric turbulence as seed perturbation of unstable growth. The compressed regions appear as thin shells, moving outward with nearly the stationary wind speed. Due to the lack of synchronization between different radial directions, the shells probably break up into fragments of small lateral extent. The space between fragments is essentially void, but at the outer side of the dense shells exist extended gas reservoirs. From their outer edge, small gas cloudlets are ablated and accelerated to high speed by radiation pressure. Propagating through an almost perfect vacuum, they catch up with the next outer shell and ram into it. In this collision, the gas parcels are heated and emit thermal X-radiation. This mechanism works within a limited radial range of the wind acceleration zone.

The two structural wind components of highly compressed, cool fragments and hot X-ray emitting gas parcels are disjunctive. X-ray emission ceases when the wind reaches its terminal speed. On the other hand, despite the expansion due to the internal pressure, the cool fragments are maintained to distances of a few tens of the stellar radius, before they gradually dissolve into a homogeneous outflow. 


\subsection{Assumptions}

Our stochastic model for X-ray line formation is designed to describe in a generic way the structures and physical conditions of the stellar wind, as summarized in the preceding subsection:

1. the flow is spherically symmetric and stationary in a statistical sense. The matter propagates according to a monotonic velocity law;

2. the initially homogeneous wind becomes fragmented at some specified distance from the stellar photosphere. The fragmentation is maintained out to a distance $r_{\mathrm{sh}}^{\max }$ at which the fragments dissolve into a homogeneous outflow. In the radial interval $\left(r_{\mathrm{sh}}^{\min }, r_{\mathrm{sh}}^{\max }\right)$ all wind material is condensed into a finite number of discrete fragments of spherical shells which propagate in radial direction;

3. all shell fragments cover the same solid angle as seen from the center of the star, and preserve this angle during their propagation. In contrast to Paper I this solid angle is not assumed to be infinitesimally small;

4. we consider different statistical distributions of the cool fragments: "concentric spheres", "broken spheres", "random fragments", and "cones". These models are described in Sect. 2.3. The statistical approach of Paper I corresponds to the "random fragments" distribution;

5. the X-ray emission originates from parcels of hot gas which are randomly distributed. Their location is confined to the radial range $r_{\mathrm{em}}^{\min }, r_{\mathrm{em}}^{\max }$. There is no self-absorption by emitting material and no re-emission of X-rays which became absorbed by cool fragments. Hence, emission is decoupled from absorption, which allows for a formal solution of the radiative transfer equation.

Besides these physical assumptions, we restrict the model to two-dimensional symmetry. Throughout the paper the index $j$ is used when addressing cool absorbing fragments, while the index $i$ refers to parcels of X-ray emitting hot gas.

\subsection{Distribution of absorbers}

We introduce Cartesian coordinates as follows. The $z$ axis points towards the observer, while the impact parameter $p$ stands perpendicular to the latter. Alternatively, a point $(p, z)$ is specified by spherical coordinates, i.e. the radius $r=\sqrt{p^{2}+z^{2}}$ and the angle $\vartheta=\cos ^{-1} \mu$ between the line of sight and the radial vector. Hence $\mu=z / r$.

For the velocity we adopt the standard " $\beta$-law"

$v(r)=v_{\infty}\left(1-\frac{r_{0}}{r}\right)^{\beta}$,

where $v_{\infty}$ is the terminal velocity, and $r_{0}$ is chosen such that $v\left(r=R_{*}\right)=0.01 v_{\infty} . R_{*}$ is the stellar (photospheric) radius. For a given mass loss rate $\dot{M}$, the continuity equation defines the density stratification via $\rho(r)=\dot{M} /\left(4 \pi r^{2} v(r)\right)$. We consider continuum absorption of X-rays by the cool wind component, caused predominantly by bound-free and K-shell ionisation processes. The opacity scales linearly with the absorber density. Assuming for simplicity that the relative population numbers of the absorbers do not vary with radius, the opacity $\chi$ scales with the mass density $\rho(r)$,

$\chi(r)=\kappa \rho(r)$

where $\kappa$ is the mass absorption coefficient which can be calculated from the chemical composition, population numbers and the continuum cross sections at the frequency of the considered $\mathrm{X}$-ray line. To avoid explicit reference to these quantities, we specify the wind by its total radial optical depth,

$\tau_{*}=\int_{R_{*}}^{\infty} \chi(r) \mathrm{d} r$.

For a homogeneous wind, the optical depth $\tau_{\mathrm{h}}(p, z)$ between a point $(p, z)$ and the observer is

$\tau_{\mathrm{h}}(p, z)=\frac{\kappa \dot{M}}{4 \pi} \int_{z}^{\infty} \frac{\mathrm{d} z^{\prime}}{r^{\prime 2} v\left(r^{\prime}\right)}$

with $r^{\prime}=\sqrt{p^{2}+z^{\prime 2}}$.

Next we proceed to the fragmented wind. We introduce different versions of our model, according to different spatial arrangements of the compressed fragments (see Fig. 1).

Model "concentric spheres". First, we consider a model where the matter is radially compressed into $N_{r}$ discrete shells forming full concentric spheres. In order that the time-averaged mass-flux $\dot{M}$ of shells resembles that of a stationary, homogeneous wind, the probability to find a shell in the radius interval $r, r+\mathrm{d} r$ must scale with $1 / v(r)$. A corresponding set of $N_{r}+1$ random radii is generated by means of the von Neumann rejection method (e.g., Press et al. 1992). All the matter located between two subsequent radii is compressed into a thin shell, moving with the stationary wind speed. The mass in each shell is conserved with time.

The total mass of a homogeneous wind enclosed between two subsequent random radii $r_{a}$ and $r_{b}$ is

$M_{j}=\int_{r_{a}}^{r_{b}} 4 \pi r^{2} \rho(r) \mathrm{d} r=\dot{M} \int_{r_{a}}^{r_{b}} \frac{\mathrm{d} r}{v(r)}$

In the fragmented wind model, this material is swept up into a thin shell of infinitesimal thickness $\Delta r$ located at $r_{j}$. The volume of this shell is $V_{j}=4 \pi r_{j}^{2} \Delta r$, its matter density is $M_{j} / V_{j}$, therefore the radial optical depth across the shell becomes

$\tau_{j}^{\mathrm{rad}}=\frac{\kappa M_{j}}{4 \pi r_{j}^{2}}=\frac{\kappa \dot{M}}{4 \pi r_{j}^{2}} \int_{r_{a}}^{r_{b}} \frac{\mathrm{d} r}{v(r)}$.

Note that the shell thickness $\Delta r$ serves only as an auxiliary variable, and cancels in the expression for $\tau_{j}$. Finally, the location of the shell, $r_{j} \in\left[r_{a}, r_{b}\right]$, is determined by the condition that this radial optical depth should be the same as for the homogeneous wind when considering the radial range from where the material has been swept up:

$\tau_{\mathrm{h}}^{\mathrm{rad}}=\int_{r_{a}}^{r_{b}} \chi(r) \mathrm{d} r=\frac{\kappa \dot{M}}{4 \pi} \int_{r_{a}}^{r_{b}} \frac{\mathrm{d} r}{r^{2} v(r)}$ 

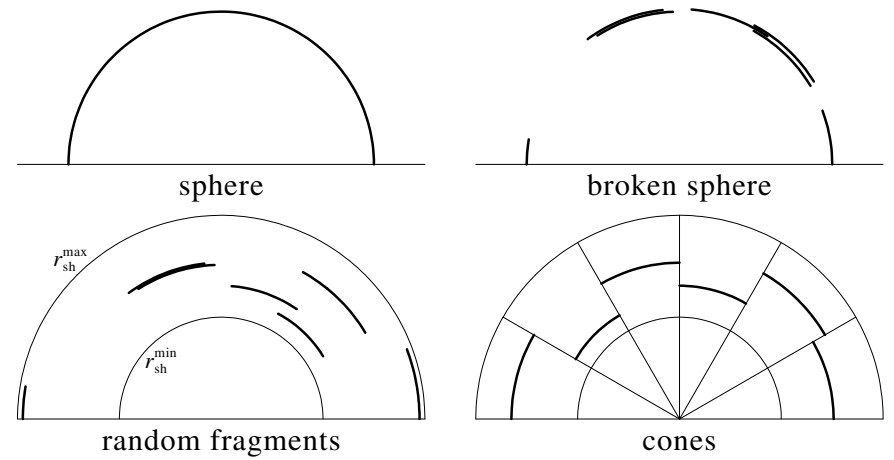

Fig. 1. Sketch of the distribution of absorbing fragments for different models, for $\left\langle N_{r}\right\rangle=1$.

Requiring $\tau_{j}^{\mathrm{rad}}=\tau_{\mathrm{h}}^{\mathrm{rad}}$ from Eqs. (6) and (7), the shell location is given by

$r_{j}^{2}=\int_{r_{a}}^{r_{b}} \frac{\mathrm{d} r}{v(r)} / \int_{r_{a}}^{r_{b}} \frac{\mathrm{d} r}{r^{2} v(r)}$.

In case of constant velocity, the shell location is just the harmonic mean between the boundaries, $r_{j}=\sqrt{r_{a} r_{b}}$.

Thus, if shells are distributed according to Eq. (8), where $r_{a}$ and $r_{b}$ are two random numbers, not only the mass loss rate is the same as for the homogeneous wind but so is the radial optical depth.

Model "broken spheres". As a next step, the absorbing spheres from the previous section are cut into pieces of equal size. In the actual 2-D cross section we consider, we cut each ring into $N_{\vartheta}$ arcs of angle $2 \pi / N_{\vartheta}$. Next, the angular positions $\vartheta_{j}$ of the arcs are randomly distributed between 0 and $2 \pi$ with uniform probability. Note that an independent set of angles $\vartheta_{j}$ is generated for each previously diced $r_{j}$. The fragments partially overlap, leaving some radial directions free.

Model "random fragments". In this model both coordinates $r_{j}$ and $\vartheta_{j}$ are chosen randomly and independent of each other. The probability distribution is uniform in radius and in angle. In the statistical limit $N_{\vartheta} \rightarrow \infty$, and assuming constant wind speed, this model was studied analytically in Paper I.

Model "cones". One might doubt whether in a real stellar wind the radial and angular distributions of fragments can be statistically independent, as assumed in the random fragment model. One expects motions to be radial with negligible transversal component. The inner, not yet fragmented zone of the wind provides a steady, homogeneous, and angle-independent mass flux. The random fragment model conserves this mass only in the statistical sense, as at any moment the number of fragments in a given radial direction may differ from that in another direction. In a snapshot of the random fragments model, the radial optical depth is therefore different in different directions. In order to investigate whether this has any puzzling consequences, we put forward an alternative model where the radial optical depth is strictly the same in all directions, as in the concentric spheres model. To achieve this, we divide the angle interval $[0,2 \pi]$ into $N_{\vartheta}$ equal segments. Then sets of $N_{r}+1$ random radii $r_{j}$ are generated independently for each of these "cones" according to the description given for the "concentric spheres", and the fragments are placed accordingly. We emphasize that each fragment is confined exactly to its cone. Therefore the radial optical depth and the mass in each radial direction is identical to that of a homogeneous wind at any instant of time.

\subsection{Distribution of emission}

The emitting gas is also distributed stochastically. We assume that a large but finite number, $N_{\mathrm{em}}$, of small parcels of emitting gas are randomly distributed in the stellar wind in the radial interval $\left(r_{\mathrm{em}}^{\min }, r_{\mathrm{em}}^{\max }\right)$. It is assumed for simplicity that the emitting zones maintain their identity during propagation with the velocity law $v(r)$; this implies that the probability to find an emitting zone in the radial interval $(r, r+\mathrm{d} r)$ scales with $v^{-1}$. We assume the same velocity law for both emission and absorption, and determine the location of emitting zones again by von Neumann's rejection method.

After the radius $r_{i}$ of an emitting parcel is selected, the random choice for $\vartheta_{i}$ is to be made. For a uniform distribution over the sphere, the probability distribution of $\vartheta$ must scale with $\sin \vartheta$. This corresponds to a uniform probability distribution of $z$ in the interval $\left(-r_{i}, r_{i}\right)$. As our model is rotationally symmetric around the $z$ axis, we put the azimuth to $\varphi_{i}=0$. Figure 2 shows one of these line-emitting zones, identified by its index $i$, and located at spherical coordinates $\left(r_{i}, \vartheta_{i}\right)$, corresponding to Cartesian coordinates $\left(z_{i}, p_{i}\right)$. Actually $\vartheta_{i}$ does not appear explicitly in our formalism, but only $r_{i}, z_{i}$ and $p_{i}=\sqrt{r_{i}^{2}-z_{i}^{2}}$ do.

A particular X-ray emitting zone with index $i$ has a line luminosity $L_{i}$. We make the following simplifying assumptions. All emitting parcels of gas contain the same amount of matter at the same temperature. The line emission is powered by collisional excitation and therefore scales with the density-squared. During motion, each zone expands according to the continuity equation. Hence, the emission $L_{i}$ of zone $i$ at radius $r_{i}$ scales with density,

$L_{i}=\frac{L_{0}}{r_{i}^{2} v\left(r_{i}\right)}$,

with a constant $L_{0}$ determined by the line emissivity and the filling factor.

The total X-ray line luminosity internally released by the wind is

$L_{\mathrm{X}, \mathrm{int}}=\sum_{i=1}^{i_{\max }} L_{i}$

Because of absorption by shell fragments, only a fraction of this radiation will emerge from the atmosphere.

\section{Radiative transfer}

After the X-ray line emitting zones and the continuum absorbing shell fragments have been specified, the radiative transfer is calculated in order to obtain the emergent $\mathrm{X}$-ray line profile. 


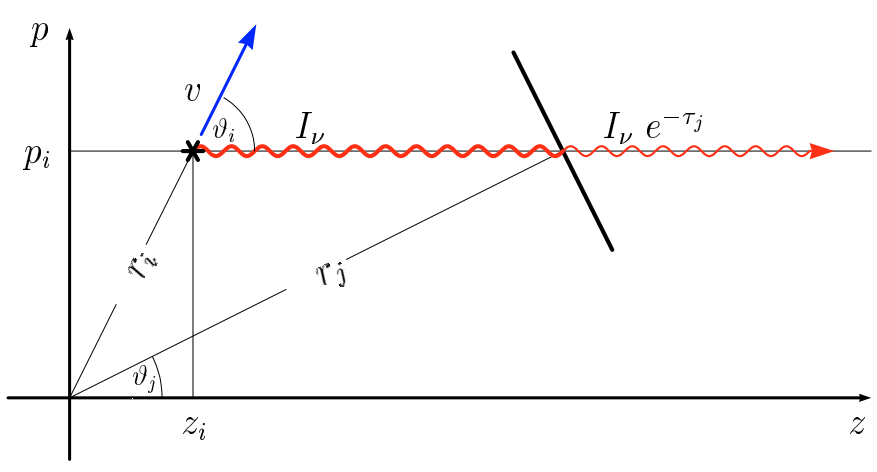

Fig. 2. Sketch of the coordinate system for the fragmented wind model. X-ray line photons are emitted by hot, small gas parcels. Shown is the emission site $i$ located at random coordinates $\left(p_{i}, z_{i}\right)$, and moving with velocity $v(r)$ in the radial direction. The cool wind consists of compressed and aligned fragments that are also randomly distributed. The radiation from parcel $i$ towards the observer at $z \rightarrow \infty$ has to cross a shell fragment $j$ at radius $r_{j}$ and covering an angle interval $\left[\vartheta_{j}-\frac{1}{2} \Delta \vartheta, \vartheta_{j}+\frac{1}{2} \Delta \vartheta\right]$. The optical depth across fragment $j$ for the ray in $z$ direction is $\tau_{j}$, reducing the line intensity $I_{v}$ by a factor $\exp \left(-\tau_{j}\right)$.

In our model emission is decoupled from absorption, which allows for the formal solution of the radiative transfer equation. Formulating the radiative transfer in the usual notation (cf. Mihalas 1978), we define the emissivity $\eta_{v}$ of the X-ray emitting zone with index $i$ as

$$
\begin{aligned}
& \eta_{v}\left(v_{\mathrm{cmf}}, p, z, \varphi\right)= \\
& \quad \frac{L_{i}}{4 \pi} \delta\left(p-p_{i}\right) \delta\left(z-z_{i}\right) \delta\left(p_{i}\left(\varphi-\varphi_{i}\right)\right) \delta\left(v_{\mathrm{cmf}}-v_{0}\right) .
\end{aligned}
$$

In order to take advantage of the rotational symmetry of our model around the $z$ axis, cylindrical coordinates $(p, z, \varphi)$ are used here. The delta functions account for the negligible spatial extension of the zone and the negligible intrinsic line width. All delta functions are normalized with respect to the integral over their argument, $\int \delta\left(p-p_{i}\right) \mathrm{d} p=1$, etc. Note that the radiation is emitted at the line center frequency $v_{0}$ as measured in the frame of reference co-moving with the flow. The corresponding frequency $v$ as seen by an observer at $z \rightarrow \infty$ is Doppler-shifted,

$v_{i, \mathrm{obs}}=v_{0}\left(1+\frac{z_{i}}{r_{i}} \frac{v\left(r_{i}\right)}{c}\right)$.

If there were no absorption, the observer would receive an intensity

$I_{v, i}(v, p, \varphi)=\frac{L_{i}}{4 \pi} \delta\left(p-p_{i}\right) \delta\left(p_{i}\left(\varphi-\varphi_{i}\right)\right) \delta\left(v-v_{i, \mathrm{obs}}\right)$

as obtained by integrating Eq. (11) over $z$.

It may happen that X-rays emitted from the considered parcel of hot gas have to cross one ore more absorbing shell fragments while traveling in the direction towards the observer. Thus we have first to find out which fragments cover the impact parameter $p_{i}$ of the emission spot. Imagine, the shell fragment with index $j$ meets this condition (cf. Fig. 2). The radial optical depth assigned to this fragment is $\tau_{j}^{\mathrm{rad}}$. If the center of this fragment has spherical coordinates $\left(r_{j}, \vartheta_{j}\right)$, the optical depth actually experienced by the X-ray is

$\tau_{j}=\frac{\tau_{j}^{\mathrm{rad}}}{\left|\mu_{j}\right|}$

This accounts for the longer geometrical path of rays which intersect the shell fragment at an inclined angle. For simplicity we apply a constant optical depth $\tau_{j}$ over the whole shell fragment, neglecting its curvature.

Let us compile a list $\mathcal{J}_{i}$ which contains the indices $j$ of those shells which are hit by the ray from emission zone $i$. Then the total optical depth for the ray from this zone, $\tau_{i}$, is the sum over all absorption shells crossed, i.e.

$\tau_{i}=\sum_{j \in \mathcal{J}_{i}} \tau_{j}$

The crucial point to understand is that the optical depth $\tau_{i}$ differs essentially from the optical depth of an individual fragment $\tau_{j}$. The optical depth across a fragment depends on the opacity $\chi(r)$ and the amount of swept-up material. In contrast, the optical depth along a specific line of sight, $\tau_{i}$, depends in the first place on the geometrical distribution of absorbing fragments, which defines the list $\mathcal{J}_{i}$ over which the sum in Eq. (15) is taken. If, for example, the fragments are located in such a way that the ray from a particular emission zone $i$ towards the observer does not cross any fragment, then $\tau_{i}=0$ for this zone, even if all fragments have $\tau_{j} \gg 1$. Vice versa, if there are many fragments of small $\tau_{j} \ll 1$ on the line of sight, the summed-up optical depth for that ray, $\tau_{i}$, can be significant.

For completeness, we have to specify the absorption of those parts of the wind which are not considered as being fragmented. First, the stellar core of radius $R_{*}$ is opaque, i.e. emission zones with $z_{i}<0$ and $p_{i}<R_{*}$ are obscured and produce no emergent intensity.

Furthermore, we assume that the radial range between $R_{*}$ and $r_{\mathrm{sh}}^{\mathrm{min}}$ is filled with homogeneous gas. Thus the ray from an emitter at $\left(p_{i}, z_{i}\right)$ towards the observer collects an additional optical depth

$\tau_{\mathrm{h}}^{\mathrm{int}}=\frac{\kappa \dot{M}}{4 \pi} \int_{z_{0}}^{z_{\mathrm{sh}}^{\mathrm{min}}} \frac{\mathrm{d} z}{r^{2} v(r)}$

when traveling though this internal sphere (cf. Eq. (4)). Here $z_{\mathrm{sh}}^{\min }=\sqrt{\left(r_{\mathrm{sh}}^{\min }\right)^{2}-p_{i}^{2}}$ and $z_{0}=\max \left(z_{i},-z_{0}\right)$.

Moreover, for radii larger than $r_{\mathrm{sh}}^{\max }$ the fragments decayed and the wind is assumed to be homogeneous again. The emission from all zones which lie further inward suffers an additional absorption

$$
\tau_{\mathrm{h}}^{\text {out }}=\frac{\kappa \dot{M}}{4 \pi} \int_{z_{\text {sh }}^{\max }}^{\infty} \frac{\mathrm{d} z}{r^{2} v(r)}
$$


with $z_{\mathrm{sh}}^{\max }=\sqrt{\left(r_{\mathrm{sh}}^{\max }\right)^{2}-p_{i}^{2}}$. Since we generally assume that $r_{\mathrm{sh}}^{\max }$ is large enough for the velocity at these distances being constant, Eq. (17) becomes

$\tau_{\mathrm{h}}^{\text {out }}=\frac{\kappa \dot{M}}{4 \pi p}\left[\frac{\pi}{2}-\tan ^{-1} \frac{z}{p}\right]$.

Therefore the optical depth along a ray between the emission zone $i$ and the observer is, in general, composed of the sum over the crossed shell fragments, $\tau_{i}$, plus contributions from the homogeneous parts of the wind inside and outside of the fragmented zone, i.e. $\tau_{\mathrm{h}}^{\mathrm{int}}$ and $\tau_{\mathrm{h}}^{\text {out }}$, respectively. Although this is fully implemented in our code, in the discussion of this paper we will refer merely to $\tau_{i}$ to keep the notations simple. With $\tau_{i}$ from now to be understood as the total optical depth between the X-ray emitting zone $i$ and the observer, the emergent intensity becomes

$I_{v, i}^{+}(\nu, p, \varphi)=I_{\nu, i}(v, p, \varphi) \exp \left(-\tau_{i}\right)$

The emergent astrophysical flux, at some reference radius $R$, is obtained by averaging the intensity over the disk of radius $R$,

$F_{v}=\frac{1}{\pi R^{2}} \int_{0}^{R} \mathrm{~d} p \int_{0}^{2 \pi} p \mathrm{~d} \varphi I_{v, i}^{+}(v, p, \varphi)$.

We insert $I_{v, i}^{+}$from Eqs. (13) and (19), and perform the integral over the coordinates. Due to the delta functions, this results in summing up the contributions from all emitting zones,

$F_{v}=\frac{1}{4 \pi^{2} R^{2}} \sum_{i=1}^{i_{\max }} L_{i} \exp \left(-\tau_{i}\right) \delta\left(v-v_{i, \mathrm{obs}}\right)$

We can calculate now the total X-ray line luminosity emerging from the star. For this purpose, one has to multiply the physical flux $\pi F_{v}$ with the surface of the reference sphere $\left(4 \pi R^{2}\right)$ and to integrate over all frequencies, giving

$L_{\mathrm{X}}=\sum_{i=1}^{i_{\max }} L_{i} \exp \left(-\tau_{i}\right)$

Note that the internally released X-ray line luminosity $L_{X \text {,int }}$ from Eq. (10) is correctly reproduced by Eq. (21) in case of zero absorption.

Due to the discrete character of the emitting parcels of gas, the emergent flux is composed of delta-function spikes at discrete frequencies. A spectrum of finite resolution $\Delta v$ is obtained by a corresponding binning. For each frequency index $k$, we compile a list $\mathcal{I}_{k}$ of those emission zones indices $i$ for which $v_{i, \text { obs }}$ falls into the frequency interval $\left(v_{k}-\frac{1}{2} \Delta v, v_{k}+\frac{1}{2} \Delta v\right)$. Then, by integrating the flux (Eq. (21)) over the frequency bin $k$, the binned flux at frequency $v_{k}$ becomes

$F\left(v_{k}\right)=\frac{1}{4 \pi^{2} R^{2} \Delta v} \sum_{i \in I_{k}} L_{i} \exp \left(-\tau_{i}\right)$.

The emergent flux scales linearly with the internally released $\mathrm{X}$-ray line luminosity, $L_{\mathrm{X} \text {,int }}$ (cf. Eq. (10)). It is illustrative to normalize the emergent flux profile with respect to the unabsorbed case. The total energy flux in the line for the unabsorbed case, obtained by setting all $\tau_{i}=0$ and integrating the previous equation over frequency, is $L_{\mathrm{X} \text {,int }} /\left(4 \pi^{2} R^{2}\right)$. Normalizing the emergent flux profile to this value, Eq. (23) becomes

$F^{*}\left(v_{k}\right)=\frac{1}{\Delta v L_{\mathrm{X}, \mathrm{int}}} \sum_{i \in I_{k}} L_{i} \exp \left(-\tau_{i}\right)$.

For convenience we will display the computed line profiles as function of dimensionless frequency $x$, measured relative to the line center and in Doppler units referring to the terminal wind velocity $v_{\infty}$

$x=\frac{v-v_{0}}{v_{0} v_{\infty} / c}$

Hence the line profile is confined between normalized frequencies -1 and 1 . Using frequency bins $\Delta x$, the normalized flux finally becomes

$F^{*}\left(x_{k}\right)=\frac{1}{\Delta x L_{\mathrm{X}, \text { int }}} \sum_{i \in \mathcal{I}_{k}} L_{i} \exp \left(-\tau_{i}\right)$.

If the emitting zones propagate with constant velocity and there is no absorption, the resulting line profile is box-shaped with $F^{*}\left(x_{k}\right)=0.5$ for $\left|x_{k}\right|<1$, so that the integral over the whole profile correctly contains all internally released X-ray line luminosity (cf. Eq. (10)).

Equation (26) yields the line profile for one specific random trial of the emitting spots and absorbing fragments. A different random trial will result in a different line profile, and a large number of trials (a few thousands, typically) is required to achieve a good statistical average. Phenomenologically, we can consider each trial as a snapshot of the wind. Typical exposure times of an X-ray observation are much larger than the dynamical time scale $R_{*} / v_{\infty}$ of the stellar wind, which corresponds to an average over many snapshots. Moreover, our model is 2-D, whereas in a 3-D wind different azimuthal angles will display statistically independent patterns of emission and absorption, whose different line profiles are also averaged in a flux measurement. Investigating the variability of X-ray lines would be interesting, but is beyond the scope of the present paper.

\section{Illustration of basic effects}

In this section we concentrate on simplified cases of wind emission and absorption to demonstrate the basic effects of fragmentation on line profiles.

Homogeneous wind. The line profiles from a homogeneous outflow are widely discussed in the literature (e.g., MacFarlane et al. 1991). Figure 3 displays the line profiles for an optically thick, homogeneous wind of constant velocity. All emission zones are placed at the same radius, $r_{i}=r_{\mathrm{em}}$, which is varied for the different profiles shown in the figure (labels). The further out the emission is located, the less absorption occurs 


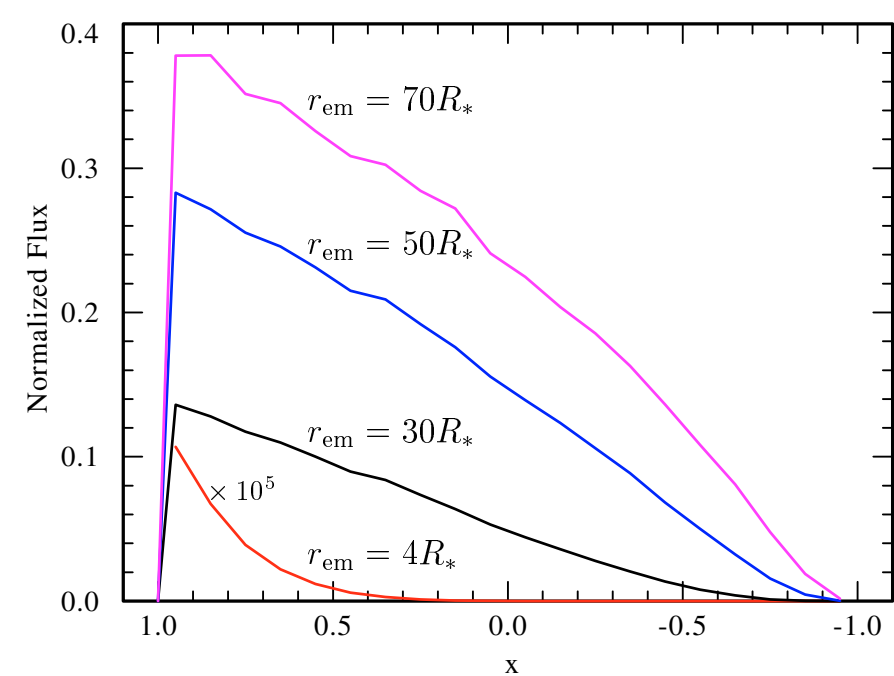

Fig. 3. Emission line profiles from a homogeneous wind of constant velocity. The radial optical depth of the photosphere is $\tau_{*}=50$ for all shown profiles. All hot gas emitting the X-ray line is assumed to be located at the same radius $r_{\mathrm{em}}$, which is varied for the different profiles shown (see labels). All profiles appear skewed and blueshifted. The lowest curve $\left(r_{\mathrm{em}}=4 R_{*}\right)$ is zoomed $10^{5}$ times.

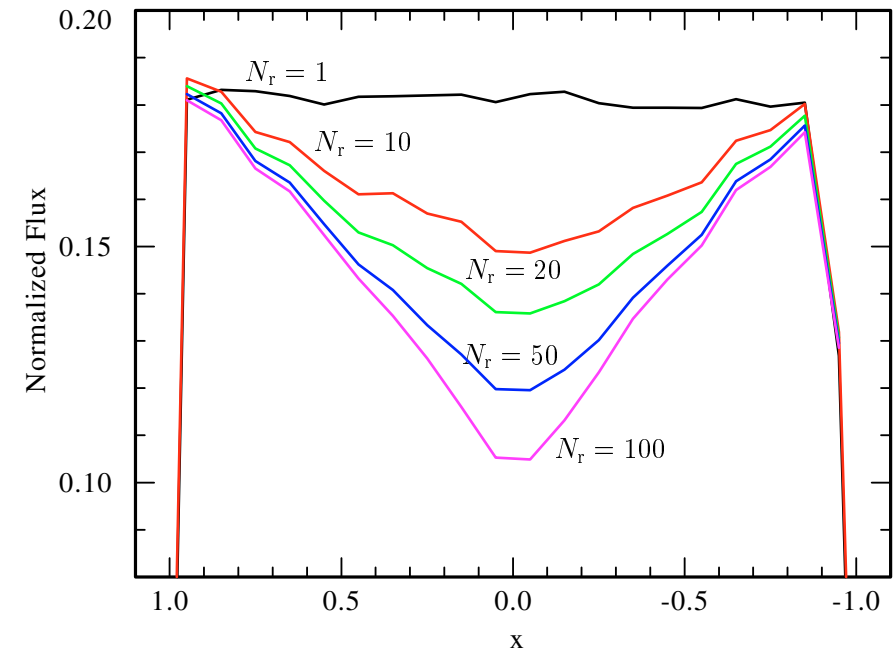

Fig. 4. Line profiles from a wind where the absorbing material is compressed in $N_{r}$ concentric shells distributed between $r_{\mathrm{sh}}^{\min }=4 R_{*}$ and $r_{\mathrm{sh}}^{\max }=100 R_{*}$. The emission originates at $r_{\mathrm{em}}=4 R *$, the wind velocity is constant $\left(v=v_{\infty}\right)$ and the total radial optical depth across all shells is $\tau_{*}=1$. The homogeneous wind below $4 R_{*}$ is omitted here. The different profiles shown correspond to different numbers $N_{r}$ of concentric spheres (labels), whose randomly chosen radii are all larger than that of the emission zone.

along rays in outward direction, i.e. in the blue part of the line profile. On the other hand, radiation from the back hemisphere which forms the red part of the line profile must always penetrate the whole wind. Therefore the resulting profiles appear skewed and blueshifted. Note that the lowest curve, corresponding to an emission radius of $4 R_{*}$, is zoomed $10^{5}$ times, which means that essentially all radiation formed deep in the wind is totally absorbed.

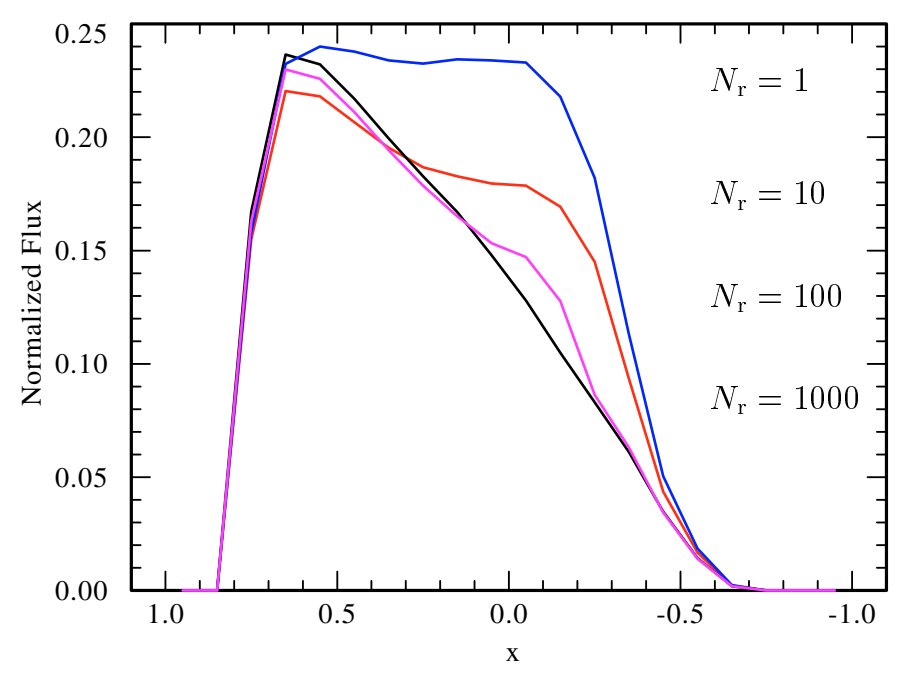

Fig. 5. The effect of a homogeneous inner wind. All parameters are the same as in Fig. 4, except that the central part of the wind between $R_{*}$ and $r_{\mathrm{em}}$ is now filled with homogeneous absorbing material. When the number of concentric spheres, $N_{r}$, becomes large, the emergent line profile converges to the line profile from a homogeneous wind of the same $\tau_{*}$. The latter is shown by the thick curve, which is indistinguishable from the case of $N_{r}=1000$ shells.

Concentric spheres. Figure 4 shows line profiles from the concentric spheres model, omitting the homogeneous inner and outer parts of the wind. Radiation emitted close to the stellar core is attenuated by an ensemble of concentric spheres located above the emission site. The location of the spheres is determined by Eq. (8). The optical depth of a shell crossed by the ray between an emitter and the observer, $\tau_{j}$, scales inversely with $|\mu|$ (Eq. (14)), i.e. depends on the intersection angle between ray and shell, and becomes large when the shell is met under a small angle. Therefore the shape of the line profile is determined by the range of angles under which the absorbing shell can be crossed by an emitted photon. For emitting spots placed at $r_{i}$ and absorbing fragments at $r_{j}$, the smallest possible value of $|\mu|$ is $\sqrt{1-\left(r_{i} / r_{j}\right)^{2}}$. Consider, for instance, the case of just one concentric shell $\left(N_{r}=1\right)$ which has swept up all material between $r_{\mathrm{sh}}^{\min }$ and $r_{\mathrm{sh}}^{\max }$, moving with constant velocity. According to Eq. (8), the shell is located at $\sqrt{r_{\mathrm{sh}}^{\min } \cdot r_{\mathrm{sh}}^{\max }}$ in order to conserve the radial optical depth. For the example shown in Fig. 4, $r_{\mathrm{sh}}^{\min }=4 R_{*}$ and $r_{\mathrm{sh}}^{\max }=100 R_{*}$, and hence the shell is located at $r_{j}=20 R_{*}$. The emission is located at $r_{i}=4 R_{*}$, i.e. at a much smaller radius. Therefore the minimum value of $|\mu|$ is close to unity, and the optical depth is nearly the same for all rays crossing the shell. Consequently, in this example with only one shell, the line profile is nearly flat-topped, the normalized flux being $F^{*}(x)=0.5 \exp \left(-\tau_{j}\right) \approx 0.5 / e$.

We increase now the number of shells $N_{r}$ into which the material is compressed. According to their random distribution, some of the shells lie closer to the minimum fragmentation radius than the one shell in the $N_{r}=1$ example. The larger $N_{r}$, the nearer is the innermost shell to the emitting sphere. For rays tangential to the emitting sphere, i.e. at line center, the intersection angle with the innermost shell becomes small. These rays 

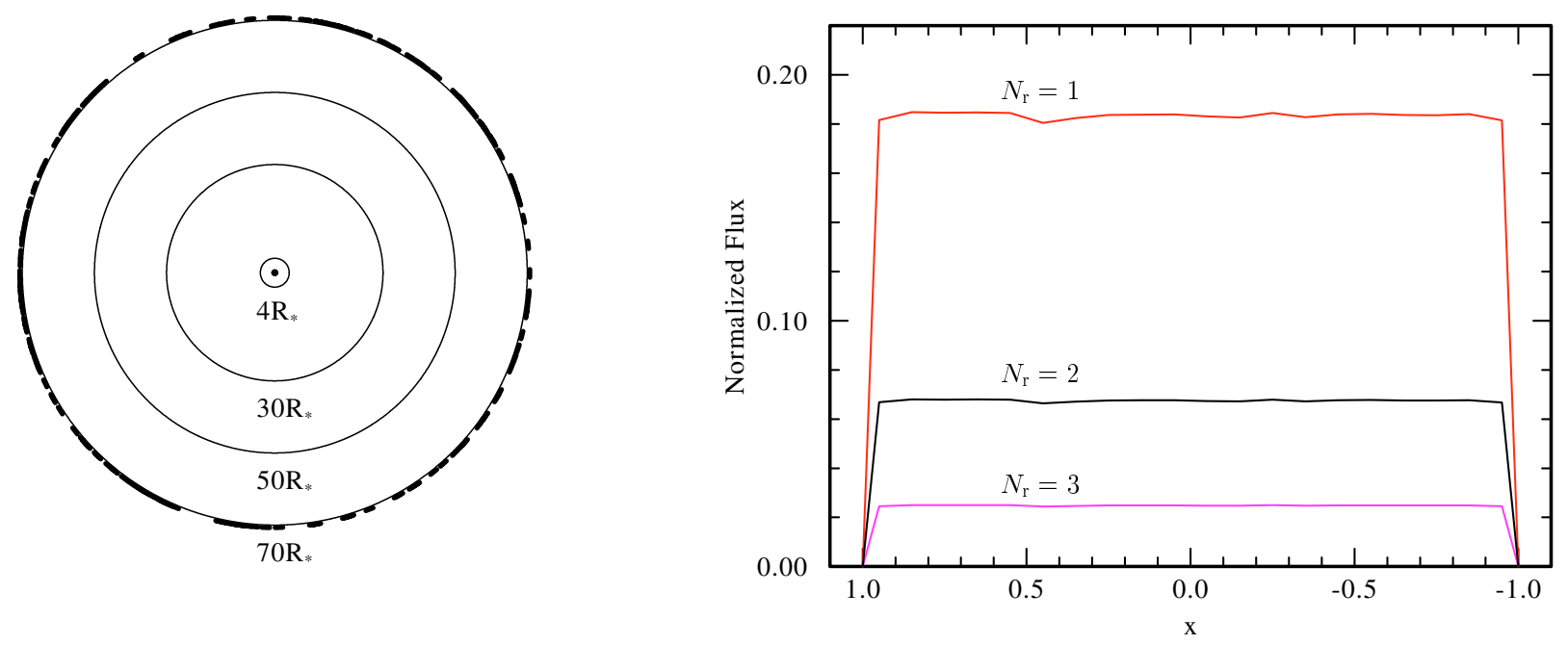

Fig. 6. The broken sphere model. One opaque sphere $\left(\tau_{*}=4\right)$ is divided into $N_{\vartheta}$ fragments, which are then randomly redistributed in angle. One of the random trials is shown in the left panel. Constant velocity is assumed. Emission arises from one sphere, for which different radii $r_{\mathrm{em}} / R_{*}=4,30,50$ and 70 are tested, but the emergent line profiles are independent from this choice. The line profiles shown in the right panel are obtained after averaging over $\approx 10^{3}$ random realizations, for the case that $N_{r}=1,2$ or 3 spheres were broken into fragments (labels).

experience strong absorption, which leads to a depression in the line center.

However, these profiles are not realistic because we have artificially omitted any absorption inside a radius of $4 R_{*}$. We now fill this region with the homogeneous inner part of the wind, while all other parameters remain as in Fig. 4. The effect is demonstrated in Fig. 5. If there is absorbing material between the stellar core and the X-ray emitting spherical layer, then radiation from the receding back hemisphere has to pass through it, whereas radiation from approaching part has not. Therefore, the red part of the line, $x>0$, is always depleted compared to the blue part, $x<0$. The blue part is flat-topped for a small number of shells, but with increasing $N_{r}$ the resulting profiles approach the shape from a homogeneous wind. This result does not depend on whether the central part of the wind is homogeneous, or arranged in a number of concentric shells.

Optically thick broken spheres. Let us start with just one absorbing shell of large optical depth, assuming that there is no absorption in the inner part of the wind, below the emission site. When all emission is formed inside absorbing shell, it will be completely trapped and the external observer will not register any emergent radiation.

In the broken sphere model, we break this absorbing shell into $N_{\vartheta}$ fragments, for simplicity all having the same size. In the 2-D cut each fragment is a fraction of a circle, i.e. an arc. These fragments are placed randomly on the circle, which means that the coordinate $\vartheta_{j}$ of the center of each fragment is a random number chosen uniformly between 0 and $2 \pi$.

Clearly, the randomly distributed fragments will overlap in some directions, and leave gaps in others. Remember that they covered one full sphere before the latter was broken. The radiation from inside the sphere can pass through these gaps entirely unattenuated.

The left panel of Fig. 6 shows one random distribution of fragments. All emission is coming from just one sphere with radius $r_{\mathrm{em}}$, for which different values are tested (labels). Constant velocity is assumed. Therefore the cosine of the angle between the direction to the observer and to an emitting spot is the normalized frequency, $\mu_{i}=x$, at which the observer receives the line photons from this spot. For one random distribution of the absorbing fragments, the optical depth along the line of sight from one specific emission spot at $\left(\mu_{i}, r_{i}\right)$ towards the observer is either zero, or big.

What is the probability that $\tau_{i}\left(\mu_{i}, r_{i}\right)=0$ ? For solid spheres, the probability that a gap occurs along a line of sight is nil. For broken spheres, simple combinatoric considerations show that, on average, a fraction $\exp (-1)$ of the sphere is free from absorbing fragments.

When a photon has a probability of $\exp (-1)$ to find a gap in the broken sphere, the emergent intensity is $I^{+}=I_{0} \exp (-1)$, always understood in the statistical limit of averaging over many random settings. In other words, the optical depth along the line of sight is unity. Note that this holds for all line frequencies, because the fractional area of gaps in a surface does not depend on the projection angle under which the surface is seen. Thus the emergent line profile is flat-topped, independent from the radius $r_{\mathrm{em}}$ of the emitting sphere (cf. Fig. 6, right panel).

It is important to realize that the flat-topped shape is due to two conditions. First, the emission must be produced in a narrow range of velocities, in order that the un-absorbed profile would be flat-topped. Second, the absorbing fragments must be thin, i.e. their radial extent must be small compared to their lateral size. Globular clouds, for instance, would not meet this condition.

So far, we considered one single broken sphere. What if there are $N_{r}$ concentric broken spheres, all located above the emission forming region? Since the $\vartheta$-randomization of fragments is independent for each sphere, the optical depth for each of them is unity, and the total optical depth adds up to $\tau_{i}=N_{r}$. 

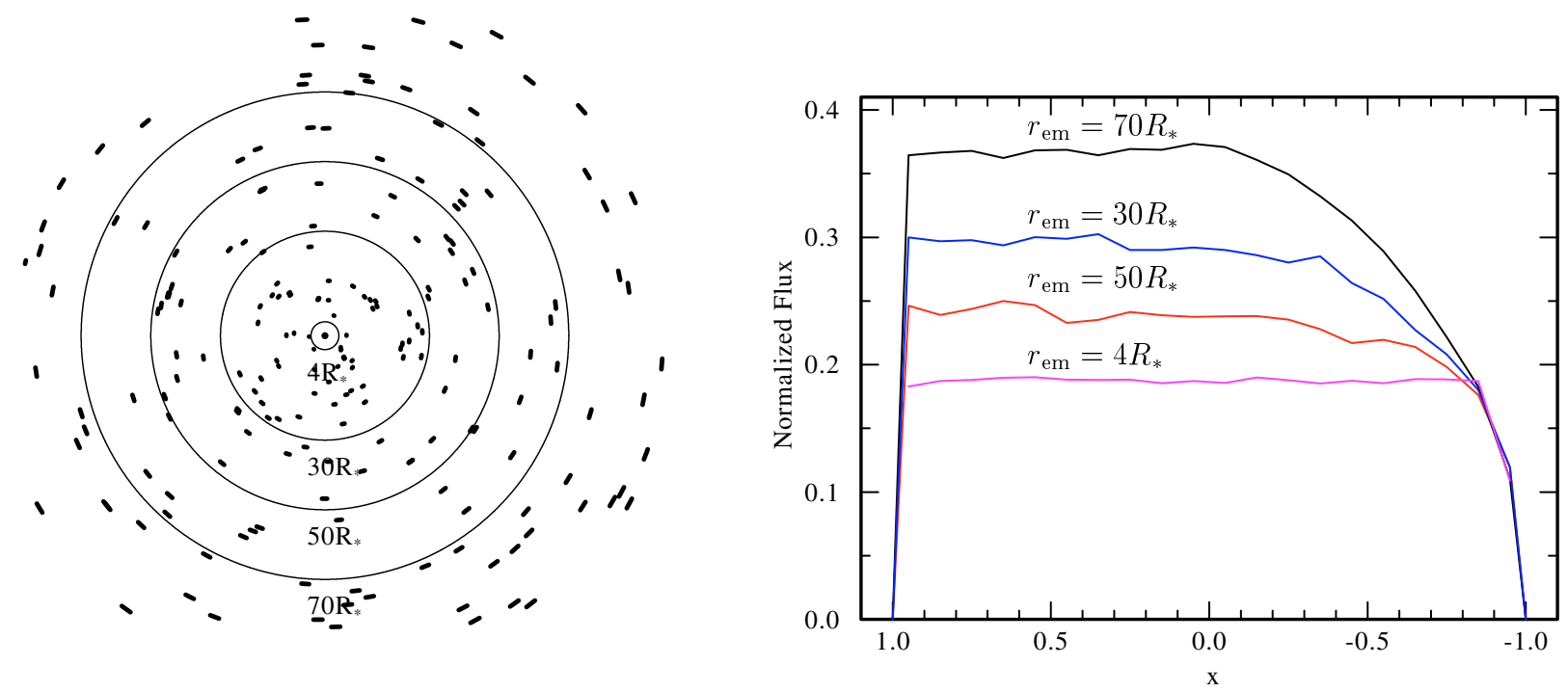

Fig. 7. Random fragments model. Left panel: one random trial of the model where the absorbing shell fragments are randomly distributed in radius (uniformly between 4.01 and $100 R_{*}$ ) and in angle. On average there is one fragment along each radial direction, $\left\langle N_{r}\right\rangle=1$. The fragments in this example are opaque, and the central part of the wind $\left(r<4 R_{*}\right)$ is void. Right panel: line profiles, for emission located at $r_{\mathrm{em}} / R_{*}=4$, 30, 50 and 70 (labels). For $r_{\mathrm{em}}=4 R_{*}$, all absorbers are outside of the emission site and the resulting profile is flat-topped as in the broken sphere model (cf. Fig. 6). If emission is located within the absorbing wind, as in cases with $r_{\mathrm{em}} / R_{*}=30,50$ and 70 , the red part of the profile is additionally absorbed in the back hemisphere, while the blue part of the line profile remains flat-topped.

The right panel of Fig. 6 shows the line profiles obtained for $N_{r}=1,2$ and 3. The left panel shows one realization of a random distribution of absorbing fragments, whereas the line profiles are accumulated over a large number of trials.

The emergent flux profiles are flat-topped. Compared to the case without any absorption $\left(F^{*}(x)=0.5\right)$, the flux is reduced by a factor $\exp \left(-N_{r}\right)$, as expected from our statistical considerations. The key difference between the line profiles in Figs. 5 and 6 is due to the fact that in the former case the transport of radiation is through the material, which leads to an angulardependent optical depth, while in the latter case the radiation emerges through the empty gaps, where the probability to find a gap does not depend on angle.

Optically thick random fragments. So far we treated first the case where the radius of the absorbing (full) concentric shells was chosen randomly; and second the case of one or more shells which were broken into fragments and distributed randomly in angle, while the fragments kept their given radial coordinate. We combine these cases now and distribute the individual fragments randomly and independently in both the angular and the radial coordinate. For the moment the fragments are assumed to be opaque.

The left panel of Fig. 7 shows one trial for this random fragments model. The total solid angle of all fragments corresponds to one full sphere. The absorbing fragments are distributed between 4.01 and $100 R_{*}$.

To start with, let us assume that the emitters are located closer to the star than any absorbing material. In Fig. 7 this corresponds to the example with $r_{\mathrm{em}}=4 R_{*}$. According to the parameters of this model, there is, after averaging over many trials, one absorbing fragment in each radial direction, $\left\langle N_{r}\right\rangle=1$.
This is exactly the same situation as in the broken sphere model considered above. Emerging radiation suffers absorption with an optical depth $\tau_{i}=1$, leading to the formation of a rectangular line profile.

Next let us consider emission which is permeated with the absorbing fragments. Again for clarity, we assume that the radiation is produced at a single radius $r_{\mathrm{em}}$. In Fig. 7 the radii of emission are chosen to be $4,30,50$, and $70 R_{*}$. When the emission takes place in outer parts of the wind, say at $70 R_{*}$, there are not many fragments left between $r_{\mathrm{em}}=70 R_{*}$ and $r_{\mathrm{sh}}^{\max }=100 R_{*}$. As can be seen from the figure, there are many gaps for photons to escape unattenuated from the front hemisphere towards the observer. Since we assume constant velocity in this example, the fragments are uniformly distributed in radial direction. Hence the average number of absorbing fragments along a radial ray from $r_{\mathrm{em}}$ to $r_{\mathrm{sh}}^{\max }$ is $\left\langle N_{r}\right\rangle\left(r_{\mathrm{sh}}^{\max }-r_{\mathrm{em}}\right) /\left(r_{\mathrm{sh}}^{\max }-r_{\mathrm{sh}}^{\min }\right)$, with $\left\langle N_{r}\right\rangle=1$ in our example. For instance, with $70 R_{*}$ for the radius of emission and $100 R_{*}$ for the outer boundary, the average number of absorbing fragments in radial direction is about 0.3 .

This number plays the role of an effective optical depth $\bar{\tau}$; the probability that there is no fragment along the radial ray from $r_{\mathrm{em}}$ to the observer is $\exp (-\bar{\tau})$. This concept will be discussed on a more general basis in Sect. 5. See also the discussion after Eq. (3) in Paper I.

For the interpretation of Fig. 7 we must understand how this effective optical depth depends on the value $\mu$ of the emitting spot located on the sphere of radius $r_{\mathrm{em}}$ (remember that the radiation from $\mu$ is observed at dimensionless frequency $x=\mu$ ). Obviously, the probability for a ray to hit a fragment depends only on the radial interval which the ray has to cross. For the front hemisphere $(\mu>0)$ this range is $r_{\mathrm{sh}}^{\max }-r_{\mathrm{em}}$, independent 

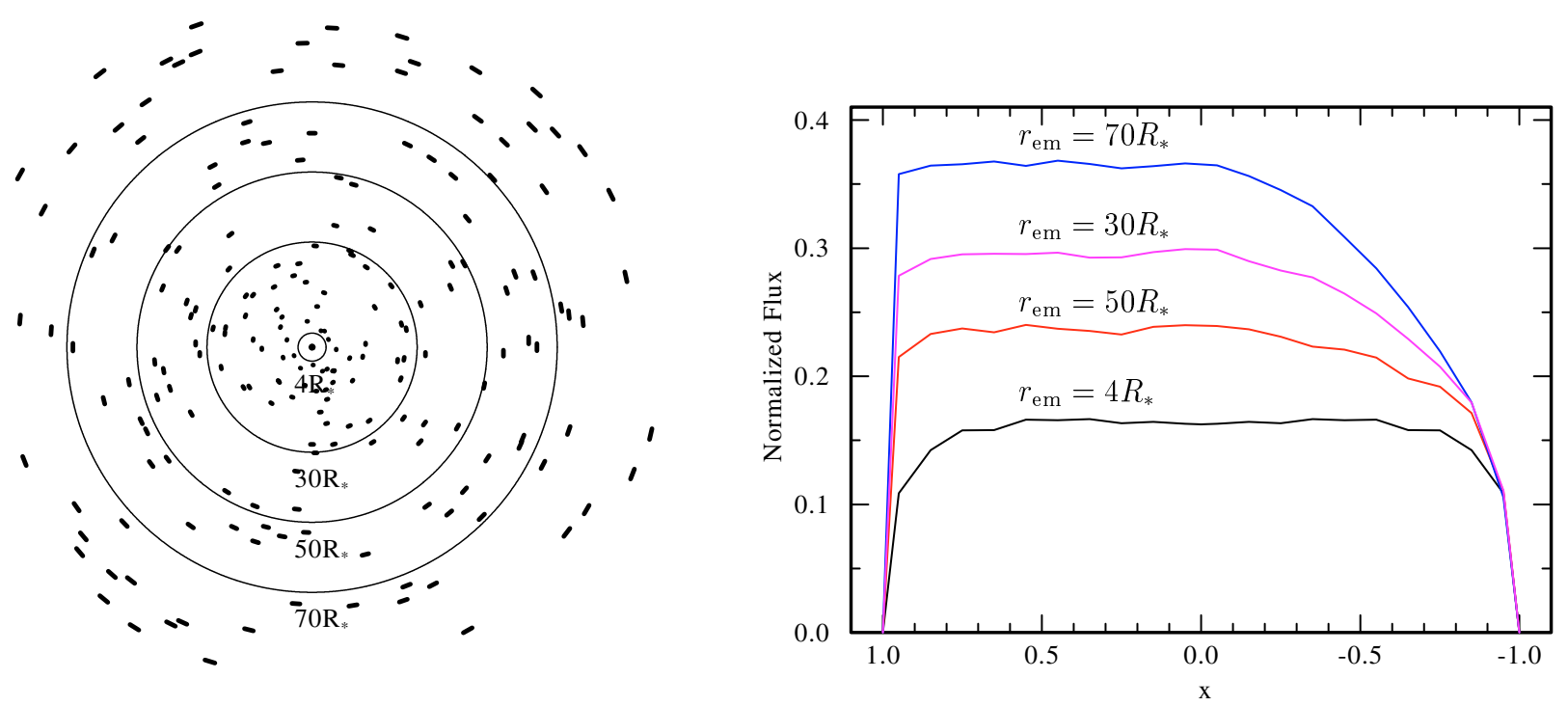

Fig. 8. Cones model. An absorbing sphere (i.e. a circle in the 2-D cross section) is divided into 200 equal arcs, which are randomly distributed in radius. In contrast to the random fragments model in Fig. 7, the fragments are kept in their angular position ("cone"). Otherwise, the model is the same as in Fig. 7. By eye one can not catch a big difference between this distribution and the one for the random fragments model. This is reflected by the line profiles shown in the right panel, which look very similar to those in Fig. 7. The overall flux is only slightly lower than in case of random fragments. The biggest difference is at the blue edge of the profile. This is due to the fact that in the cones model the radial optical depth is preserved (here, $\tau_{*}=4$ ), and photons emitted in almost radial direction towards the observer cannot find gaps to escape.

of $\mu$. Therefore the blue part of the profiles in Fig. 7 is flattopped, and the flux level is $\exp (-\bar{\tau})$ times the the flux of the un-absorbed emission.

If the emitters are located at $r_{\mathrm{em}}=4 R_{*}$, the flat-topped shape extends also to the red side of the profile, because of our somewhat artificial assumption in this example that the central part of the wind $\left(r<4 R_{*}\right)$ is void. However, when the emitting spots are permeated with the fragments (say, at $r_{\mathrm{em}}=50 R_{*}$ ), a ray from an emitting spot on the back hemisphere $(\mu<0)$ with coordinates $\left(p_{i}, z_{i}<0\right)$ collects optical depth from both hemispheres. In radius, the ray first descends from $r_{\mathrm{em}}$ to $r=$ $p_{i}$ (back hemisphere), and than climbs from $r=p_{i}$ to $r_{\mathrm{sh}}^{\max }$ (front hemisphere). Thus the total radial range crossed is $r_{\mathrm{sh}}^{\max }+$ $r_{\mathrm{em}}-2 p_{i}$, and the optical depth is proportional to this range. Therefore the red part of the line becomes strongly absorbed and skewed.

Cones. This model was introduced in Sect. 2.3. Each absorbing sphere is divided into $N_{\vartheta}$ segments (i.e. arcs in the twodimensional cross section), which are randomly distributed in radius. In contrast to the random fragments model, the fragments are kept at their angular position ("cone"), and not randomized in lateral direction. This model is interesting, because the radial optical depth and the mass are conserved for each radial direction. One of the random trials for the distribution of absorbing fragments is shown in the left panel of Fig. 8. As in the previous examples, the emission is located at a unique radius $r_{\mathrm{em}}$, and there is no absorption below this radius. As seen by eye, this distribution of the fragments can hardly be distinguished from the one in Fig. 7.

The resulting line profiles are shown in the right panel of Fig. 8. They also do not differ much from the line profiles obtained in the random fragment case. The overall flux is only slightly lower. The biggest difference is at the blue edge of the profiles. This is due to the fact that in the cones model the radial optical depth is conserved (here, $\tau_{*}=4$ ), and photons emitted in almost radial direction towards the observer cannot find gaps to escape from absorption. But apart from the blue edge, the radial randomization of the absorbing fragments provides escape channels for the photons, acting in the same way as in the random fragments model.

It order to verify this numerical result for 3-D, we made numerical experiments to illustrate the effect of random radial re-positioning of fragments of a sphere. We cover the unit sphere with spherical triangles of similar size, mimicking absorber fragments. Spherical coordinate lines are not appropriate here, due to the dependence of solid angle on $\sin \vartheta$. The sphere is divided into octants. For each of the three grand circle segments delimiting an octant surface, the midpoint at half arc length is connected to the midpoints of the other two grand circle segments. This divides the octant surface into four spherical triangles. For each of them this procedure (division of grand circle segments at half arc length, and connection to nearest neighbors) is repeated. After six such subdivisions one obtains $4^{7}=16384$ triangles on the front hemisphere. These triangles are of similar size and shape, but not exactly uniform.

Next, the radial location of these (opaque) triangles is chosen randomly and uniformly between $r=0.8$ and 1 . The corresponding sky projection is shown in Fig. 9. In fact, the distribution appears random and uniform, except in the disc center and close to the limb. To quantify this, we divide the projected disk into 10 rings of equal radial extent and determine numerically the fraction of the ring area which is covered by at least one fragment (Fig. 10). The complement is the fractional area 


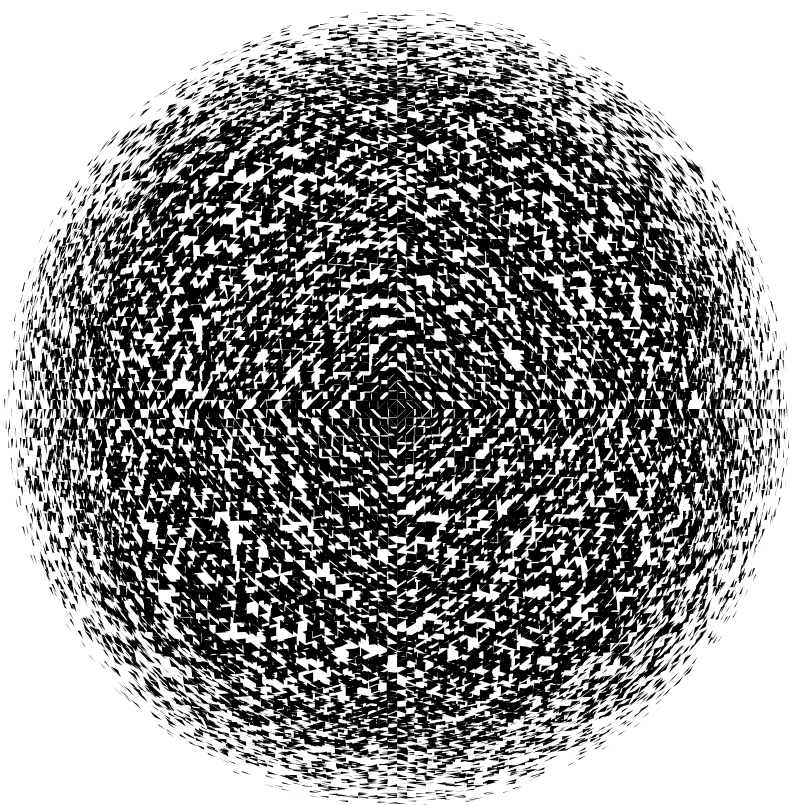

Fig. 9. Sky projection of radially randomized fragments. The front hemisphere of a unit sphere is cut into 16384 roughly equal spherical triangles, which are subsequently randomly redistributed between $r=0.8$ and 1.0 while their angular position is kept.

which remains free of any projected fragment, while fragments are overlapping at other locations. For central rays $p \ll 1$, radial randomization cannot move fragments out of the line of sight. Hence, the central region $r \leq 0.2$ remains largely obscured (small fragment overlap), $\tau>1$. For $0.2 \leq r \leq 0.8$, the transmission is close to $1-e^{-1}$, as expected if the fragments were randomly distributed laterally on the surface of the sphere. For $r>0.8$ finally, $\tau<1$ because fragments are radially distributed over this range. To summarize, for an observer at infinity, radial randomization in a spherically symmetric wind is largely indistinguishable from lateral randomization.

\section{Effective opacity}

An alternative approach to the stochastic numerical modeling of this paper is to introduce an effective optical depth $\bar{\tau}_{i}$ for a random distribution of absorbing fragments by statistical means (cf. Paper I). Both the numerical and the analytical approach should yield identical results for the same parameters. In this section we concentrate on the analytical description, and compare the resulting line profiles with those obtained in the numerical simulations.

Let us define the effective optical depth $\bar{\tau}_{i}$ in the conventional way, as integral over an effective opacity $\alpha$ (dimension: per length) along the path,

$\bar{\tau}_{i}=\int_{z_{i}}^{z_{\mathrm{sh}}^{\max }} \alpha \mathrm{d} z$.

The radiative transfer equation keeps its usual form

$d I_{v}=-\alpha I_{v} \mathrm{~d} z$

with the well-known solution Eq. (19).

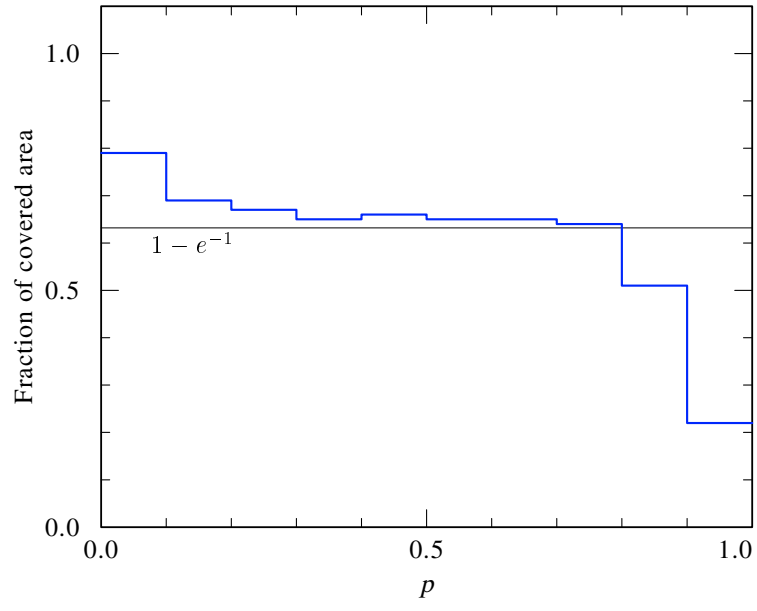

Fig. 10. Fraction of projected surface covered by radially randomized fragments. The sky projection of the fragmented hemisphere from Fig. 9 is divided into 10 concentric rings of equal width in impact parameter $p$, and the fraction of covered (black) surface is determined numerically. Except for the center and the limb region, the coverage is $\approx 1-e^{-1}$, i.e. close to the value expected if the fragments were randomly distributed in their angular position, but kept on the unit sphere.

The effective opacity is the product of the number density of absorbers and the (average) area of the absorbing fragments projected on a plane perpendicular to the line of sight. Moreover, we allow (in contrast to the usual definition of atomic cross sections) for absorbers that are not completely opaque, but have a probability, $\mathcal{P}$, that a photon hitting the cross section is absorbed.

The average number of shells within a unit radius is termed $n(r)$. In case of constant velocity, $n(r)=\left\langle N_{r}\right\rangle /\left(r_{\mathrm{sh}}^{\max }-\right.$ $\left.r_{\mathrm{sh}}^{\mathrm{min}}\right)$ is also constant. With variable velocity, the radial density of shells scales with $v^{-1}$ (cf. Sect. 2.3). Therefore we have

$n(r)=\frac{n_{0}}{v(r)}$

with

$n_{0}=\left\langle N_{r}\right\rangle \mid \int_{r_{\mathrm{sh}}^{\min }}^{r_{\mathrm{sh}}^{\max }} \frac{\mathrm{d} r}{v(r)}$.

As can be seen from Fig. 2, the area of a fragment projected to the plane perpendicular to the line of sight scales with $|\mu|$. The total area of all absorbers within a spherical layer of radius $r$ and thickness $\mathrm{d} r$ is $n(r)|\mu| 4 \pi r^{2} \mathrm{~d} r$, while the volume itself is $4 \pi r^{2} \mathrm{~d} r$. Thus the effective opacity is

$\alpha(r, \mu)=n(r)|\mu| \mathcal{P}$.

Note that this opacity is not isotropic as usual, but angulardependent. The probability $\mathcal{P}$ that a photon which encounters a fragment is absorbed is

$\mathcal{P}=1-\mathrm{e}^{-\tau_{j}}$,

i.e. is a function of the optical depth $\tau_{j}$ of fragments at $r$ with orientation $\mu$ as given by Eq. (14). 
As long as one avoids integrating through the point where $\mu=0$, the path element along the ray, $\mathrm{d} z$, can be substituted by $\mathrm{d} r$, using

$\mathrm{d} z=\mu^{-1} \mathrm{~d} r$.

By inserting Eqs. (31)-(33) into Eq. (27) we obtain for the effective optical depth between an emitting point at $\left(r_{i}, \mu_{i}\right)$ and the observer

$\bar{\tau}_{i}\left(r_{i}, \mu_{i}\right)=\int_{r_{i}}^{r_{\mathrm{sh}}^{\max }} n(r)\left(1-\mathrm{e}^{-\tau_{j}}\right) \mathrm{d} r$.

Remarkably, in contrast to Eq. (4) neither $\dot{M}$ nor $\kappa$ appears explicitly in this expression for $\bar{\tau}_{i}$. The effective optical depth is determined essentially by the geometrical distribution of fragments, as explained earlier.

In the following we will consider the limits of optically thick and optically thin fragments, $\tau_{j} \gg 1$ and $\tau_{j} \ll 1$.

\subsection{Optically thick case $\left(\tau_{j} \gg 1\right)$}

If all cool material is assembled in a number of optically thick fragments, the emergent radiation is only due to the presence of gaps between the fragment. The radiation passes through these gaps entirely unattenuated, but all photons which intersect a fragment are absorbed. Therefore the probability $\mathcal{P}$ is unity. In case of constant velocity, the integral in Eq. (34) is taken over the constant $n(r)$ and reduces to (see Eq. (4) in Paper I)

$\bar{\tau}_{i}\left(r_{i}, \mu_{i}\right)=\frac{\left\langle N_{r}\right\rangle}{r_{\mathrm{sh}}^{\max }-r_{\mathrm{sh}}^{\min }} \begin{cases}r_{\mathrm{sh}}^{\max }-r_{i} & \text { if } \mu_{i}>0 \\ r_{\mathrm{sh}}^{\max }+r_{i}-2 p_{i} & \text { if } \mu_{i}<0 .\end{cases}$

The line profiles from our stochastic numerical model shown in Fig. 7 agree extremely well with this analytical result.

\subsection{Optically thin case $\left(\tau_{j} \ll 1\right)$}

The optical depth of individual absorbers may be small because the total optical depth of the wind, $\tau_{*}$, is small, or because the optical depth is distributed over very many fragments (i.e. $N_{r}$ is large). If the absorbing fragments are optically thin, the emergent flux is both due to radiation which passed unattenuated through gaps, and to radiation which crossed fragments and was only partly absorbed.

For small optical depths, $\tau_{j} \ll 1$, the exponent in Eq. (32) can be expanded as $\exp \left(-\tau_{j}\right) \approx 1-\tau_{j}$. Consequently $\mathcal{P} \approx \tau_{j}$ and Eq. (31) simplifies to

$\alpha(r, \mu)=n(r)|\mu| \tau_{j}$.

Expressing the directional optical depth of the fragment, $\tau_{j}$, by its radial optical depth $\tau_{j}^{\mathrm{rad}}$ (Eq. (14)) yields

$\alpha(r, \mu)=n(r) \tau_{j}^{\mathrm{rad}}$.

Therefore, in the limit of optically thin fragments the effective opacity becomes isotropic, as the usual opacity in homogeneous winds.

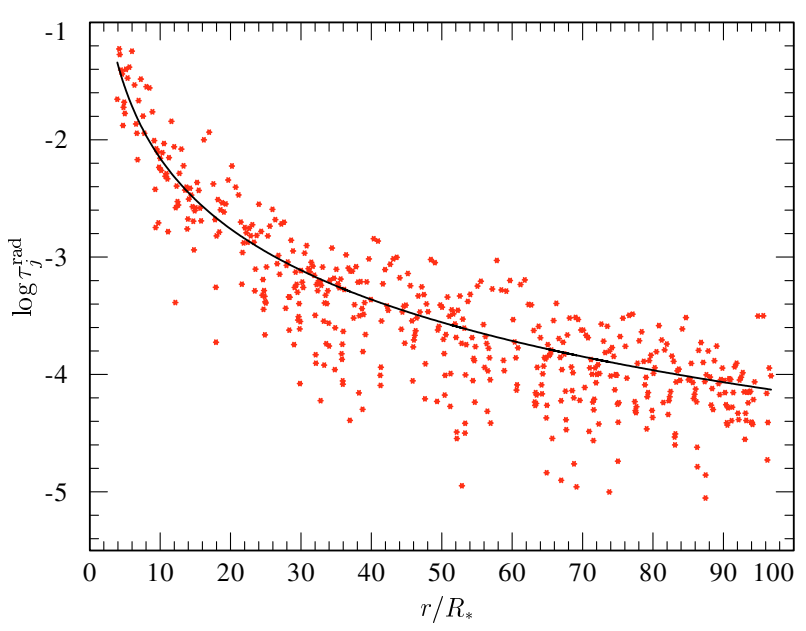

Fig. 11. Radial optical depth of individual fragments. For one selected cone in the cones model, the total radial optical depth $\tau_{*}=1$ splits into $N_{r}=500$ shells located at random radii $r_{j}$. The optical depth $\tau_{j}^{\mathrm{rad}}$ of each fragment varies according to the random algorithm used to define the mass in each shell, and is denoted by dots. The continuous line gives the average radial optical depth $\bar{\tau}_{j}^{\text {rad }}$ of the shells, if the mass is uniformly distributed over all fragments. Further parameters of the model are $r_{\mathrm{sh}}^{\min }=4 R_{*}, r_{\mathrm{sh}}^{\max }=100 R_{*}$, and $\beta=1$ for the velocity-law exponent.

We insert now for $\tau_{j}^{\mathrm{rad}}$ the average optical depth of a fragment $\bar{\tau}_{j}(r)$ at radius $r$. Remember that in our stochastic wind model the amount of matter swept up into an individual fragment is a random number. This is illustrated in Fig. 11, which shows for one cone of the cones model how the total radial optical depth $\tau_{*}=1$ splits into $N_{r}=500$ shells located at their individual radii $r_{j}$. To define an average optical depth, each shell shall have the same amount of mass. According to the geometrical dilution, the radial optical depth of the shells scales with $r^{-2}$, so that we can write

$\bar{\tau}_{j}(r)=\frac{\tau_{0}}{r^{2}}$.

The radial integral over the effective opacity must yield the total optical depth $\tau_{*}$, i.e.

$\int_{r_{\mathrm{sh}}^{\min }}^{r_{\mathrm{sh}}^{\max }} n(r) \bar{\tau}_{j}(r) \mathrm{d} r=\tau_{*}$,

which leads with Eq. (29) to

$n_{0} \tau_{0} \int_{r_{\mathrm{sh}}^{\min }}^{r_{\mathrm{sh}}^{\max }} \frac{\mathrm{d} r}{r^{2} v(r)}=\tau_{*}=\frac{\kappa \dot{M}}{4 \pi n_{0}} \int_{r_{\mathrm{sh}}^{\min }}^{r_{\mathrm{sh}}^{\max }} \frac{\mathrm{d} r}{r^{2} v(r)}$

where on the right-hand side we expressed the radial optical depth using its original definition for a homogeneous wind. Thus we have $\tau_{0}=\kappa \dot{M} /\left(4 \pi n_{0}\right)$. The continuous line in Fig. 11 shows the run of the average optical thickness of fragments as function of radius (Eq. (38)). The effective opacity in Eq. (37) for the optically thin limit finally becomes

$\alpha(r, \mu)=\frac{n_{0}}{v(r)} \frac{\tau_{0}}{r^{2}}=\frac{\kappa \dot{M}}{4 \pi} \frac{1}{r^{2} v(r)}$, 


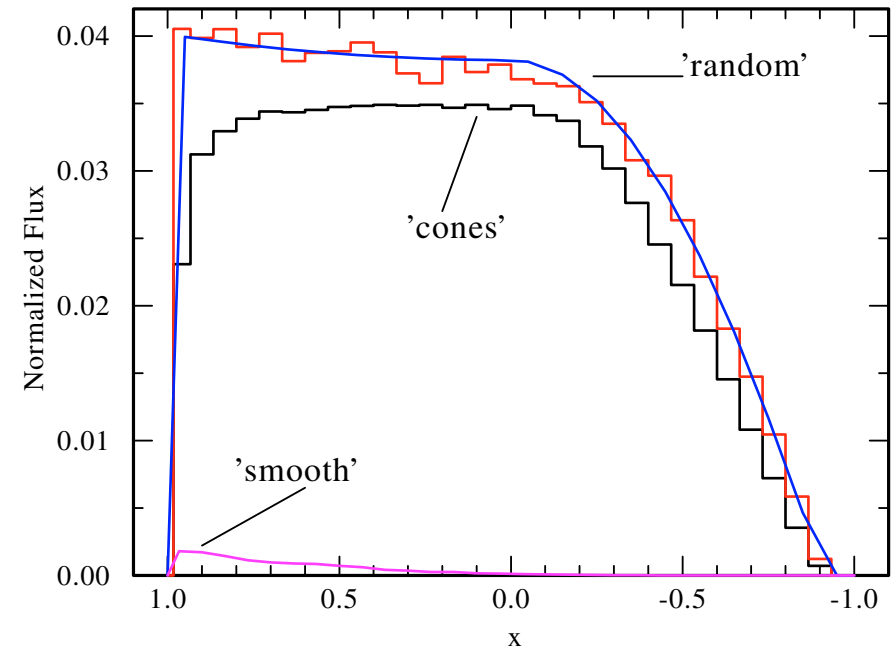

Fig. 12. Line profiles for a model with total optical depth $\tau_{*}=10$, and $N_{r}=10$ shells in radial direction. The histogram-style profiles are for the "random fragments" and the "cones" versions of our stochastic wind model. The smooth line is analytically calculated with the effective opacity from Eq. (31), and is equivalent to the statistical model in Paper I. It agrees precisely with the random fragment stochastic model. The more regular arrangement of the absorbing fragments in the cones model leads to a slightly lower emergent flux. In drastic contrast, a homogeneous wind with the same massloss rate absorbs the X-ray line almost totally (dashed line). Further model parameters are: constant velocity; emission distributed between $r_{\mathrm{em}}^{\min }=10 R_{*}$ and $r_{\mathrm{em}}^{\max }=30 R_{*}$; absorbing shells between $r_{\mathrm{sh}}^{\min }=10 R_{*}$ and $r_{\mathrm{sh}}^{\max }=100 R_{*}$.

which is identical to the opacity $\chi$ of a homogeneous wind given by Eq. (2).

To summarize, we have demonstrated that if the optical depth across all fragments is small, $\tau_{j} \ll 1$, the effective opacity in the fragmented wind is identical to the opacity of a homogeneous wind of otherwise the same parameters. We may conclude that the homogeneous wind is the limiting case of a fragmented wind, when the radial number of fragments is big and/or the total optical depth is small.

\subsection{Comparison with the analytical statistical model}

Solving the radiative transfer equation with the effective opacity from Eq. (31) is equivalent to our statistical approach in Paper I. In this subsection we discuss how this analytical treatment compares with the stochastic model constructed in the present paper.

Some resulting line profiles are shown in Figs. 12 and 13. The total optical depth is $\tau_{*}=10$ for both figures. The only difference is the number of fragments in radial direction, which is $N_{r}=10$ in Fig. 12 and $N_{r}=100$ in Fig. 13. The histogramstyle profiles are for the stochastic wind model in the "random fragments" and "cones" version. The smooth line is calculated analytically using the effective opacity from Eq. (31), and is equivalent to that from the statistical approach of Paper I. It is in excellent agreement with the random fragment stochastic model, confirming its prediction of a flat-topped, blueshifted

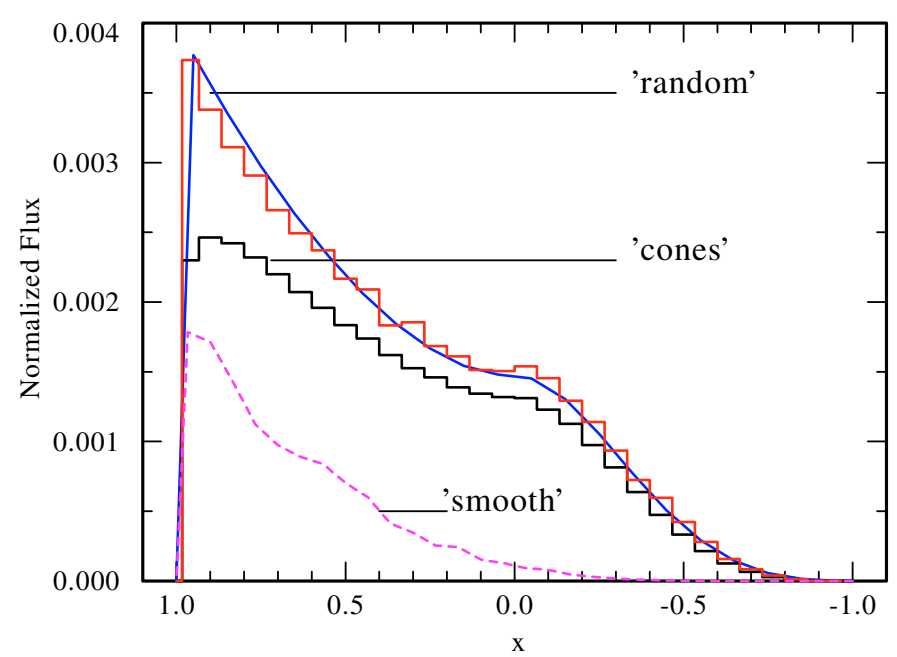

Fig. 13. Same as Fig. 12, but with $N_{r}=100$ fragments in radial direction. Due to their larger number, most of the fragments are now optically thin. Therefore the profiles from the stochastic model are more similar to that from a homogeneous wind, although still stronger by a factor of two.

and nearly symmetric profile. The more regular arrangement of the absorbing fragments in the cones model leads to a slightly lower emergent flux, especially at the blue edge of the profile.

For the parameters in Fig. 12, most fragments are optically thick. Therefore the gain in emergent flux achieved in the stochastic wind model is dramatic. A homogeneous wind with the same mass-loss rate absorbs the X-ray line almost totally (dashed line in Fig. 12), and the tiny remaining line has a triangular profile.

Figure 13 shows the result for the same models, but with the number of fragments in radial direction being increased to $N_{r}=100$. Now most of the fragments are no longer optically thick. Therefore the emergent line profile from the stochastic wind model (both versions) have a triangular shape like that from the homogeneous wind, and the gain in emergent flux is only a factor of two.

Thus our numerical results from the stochastic wind model confirm fully the predictions from the statistical considerations in Paper I. If the wind matter is compressed into a small number of optically thick shells, and these shells break up into fragments, the effective optical depth of the wind is small, independent of the amount of mass contained in the fragments. On the contrary, a homogeneous wind with the same mass-loss rate may have an arbitrarily large optical depth. Therefore the gain in emergent intensity of an X-ray line emitted from deep inside the wind may be huge, comparing the fragmented with the homogeneous model. The transparency of the fragmented wind increases with decreasing number of fragments in radial direction.

We emphasize that the line profiles from the fractured wind do not depend on the angular size of the shell fragments. The number $N_{\vartheta}$ of arcs in our 2-D cut of the model does not enter the effective opacity. The statistical limit of a smooth line profile can be achieved by a large $N_{\vartheta}$, but also by a large number of random settings over which the results are averaged. Only 


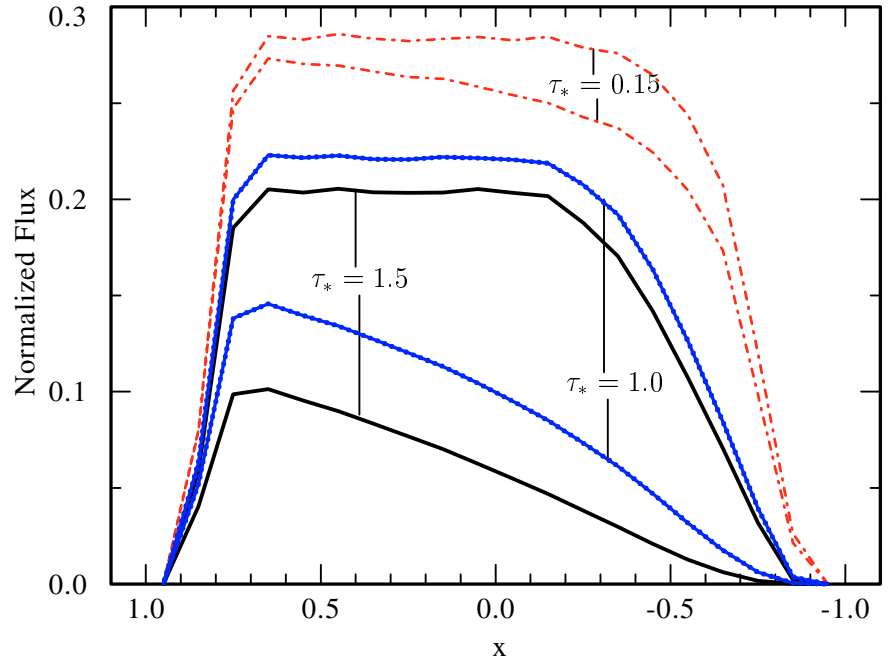

Fig. 14. Line profiles for relatively thin winds. The result from the fragmented wind is compared to the corresponding homogeneous model with the same mass-loss rate. The pairs are labeled with the total optical depth of the homogeneous wind, $\tau_{*}$. The stronger line of each pair refers to the fractured wind. The radial number of fragments is always $\left\langle N_{r}\right\rangle=1$. The differences between the fractured and the homogeneous wind become significant when $\tau_{*} \gtrsim 1$. The profiles from homogeneous models become weaker and skewed, while the fragmented model profiles remain flat-topped in the blue part. The velocity law exponent $\beta=1$.

for technical reasons, the fragments should be small enough to justify the neglect of curvature in our description. Moreover, in the cones model, too big fragments in fixed angular positions would spoil the frequency resolution and imprint spurious steps to the emergent profiles.

\section{Parameter study}

In Sect. 4 we employed simplified models in order to illustrate and understand the effects of wind fragmentation. In this section line profiles are presented for realistic wind parameters, calculated with our stochastic wind model in the "random fragments" version.

A stellar wind is specified by a few fundamental parameters, such as the stellar luminosity, the photospheric radius, the mass-loss rate, the chemical composition and the velocity law. Even for a homogeneous model, the calculation of the continuous opacity at a given X-ray frequency is not straightforward and requires in principle a Non-LTE atmospheric model. Then one can calculate the radial optical depth $\tau_{*}$ of the homogeneous model, as defined by Eq. (3). As stated already in Sect. 2.3, we assume for simplicity that the X-ray opacities scale linearly with density, and adopt $\tau_{*}$ of the corresponding homogeneous model as the free parameter of our calculations.

The spatial structure of the inhomogeneous wind depends on the radial distribution of the wind fragments and of the $\mathrm{X}$-ray line emitting spots. Important is the number of fragments in radial direction, $N_{r}$. Existing hydrodynamical simulations trace the fragmentation to as far as hundreds of stellar radii.

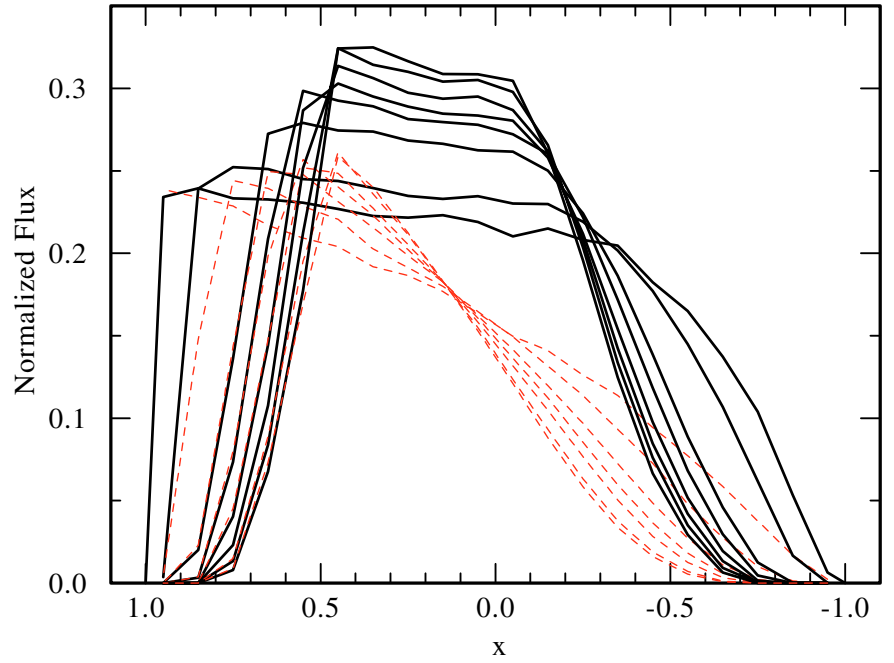

Fig. 15. Line profiles emerging from fragmented winds (solid lines) and from smooth winds (dashed lines). All models are with $\tau_{*}=1$ and $\left\langle N_{r}\right\rangle=10$. The different profiles refer to different velocity-law exponents $\beta=0,0.5,0.8,1,1.5,2,2.5,3,3.5$, and 4 , where the higher $\beta$ values yield narrower profiles.

$\mathrm{X}$-ray emission is predicted to take place only in the wind acceleration zone, i.e. out to a few stellar radii.

To keep the discussion transparent, we adopt the following parameters for all figures in this section: a smooth wind between $1 R_{*}$ and $4 R_{*}$, fragmentation of the wind between $4 R_{*}$ and $100 R_{*}$ followed by a smooth wind again further out; the $\mathrm{X}$-ray line is emitted between $4 R_{*}$ and $10 R_{*}$. The remaining parameters for the stochastic fragmented wind model are the angular extension of the fragments and the number of fragments per radius. The number of fragments in angular direction, for which we generally use $N_{\vartheta}=360$, is of no significance, as discussed in the previous section.

Therefore, when $\tau_{*}$ is specified, the most influential parameter of the model which is not constrained by independent means is the number of fragments per radius, $\left\langle N_{r}\right\rangle$. This parameter defines whether the fragments are optically thick or thin for the given mass-loss rate.

It was shown in Sect. 5.2 that fragmentation becomes effective in reducing opacity when fragments are optically thick, but cannot influence the radiative transfer in optically thin winds even if the number of fragments per radial direction, $N_{r}$, is small. To check the parameter range we calculated some models with $\left\langle N_{r}\right\rangle=1$ and different values of $\tau_{*}$ around unity. The profiles in Fig. 14 confirm that the difference between the homogeneous and fragmented wind is small for the optically thin case $\tau_{*}=0.15$, and only becomes significant when $\tau_{*} \gtrsim 1$. With increasing $\tau_{*}$ the profiles from the homogeneous models become weaker and skewed, while the fragmented model profiles remain strong and flat-topped in the blue part.

The next two figures study the influence of different velocity laws on the line profile. First we consider a relatively weak wind, with optical depth $\tau_{*}=1$. The fragmented wind is calculated with $\left\langle N_{r}\right\rangle=10$ shells in radial direction. The line profiles 


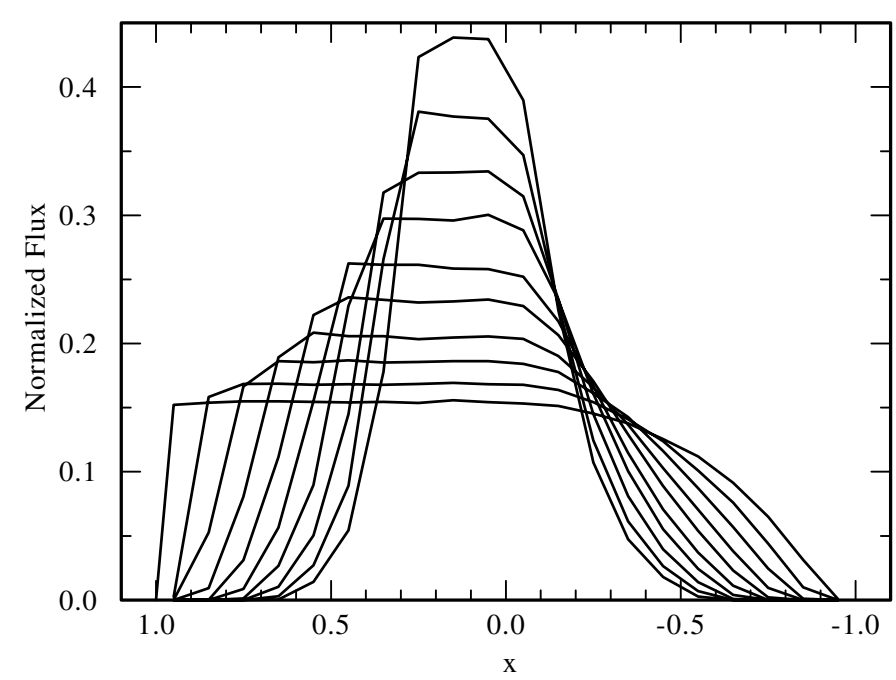

Fig. 16. Line profiles emerging from an optically thick wind, $\tau_{*}=10$, compressed into one fragment per radial direction, $\left\langle N_{r}\right\rangle=1$. The line profiles are for different velocity laws, namely for constant velocity, and exponents $\beta=0.5,1,1.5,2,2.5,3,3.5,4,4.5$ (from lower to higher maximum).

shown in Fig. 15 are for different values of $\beta=0.5,0.8,1$, $1.5,2,2.5,3,3.5$ and 4 , respectively. The broadest profile is for the constant velocity case, which can be considered as the limit $\beta \rightarrow 0$ when $v(r)$ jumps to $v_{\infty}$ immediately at the stellar surface. The higher $\beta$, the slower is the wind acceleration. CAK-type hydrodynamic modeling of $\mathrm{O}$-star winds arrives at $\beta \approx 0.8$, typically, while certain empirical facts have been interpreted in favor of higher $\beta$ values. The X-ray line profiles from our stochastic wind model show some sensitivity to this parameter.

At the opposite extreme, Fig. 16 shows an optically thick wind $\left(\tau_{*}=10\right)$ compressed into just one fragment per radial direction $\left\langle N_{r}\right\rangle=1$. The line profiles are again for different velocity-law exponents $\beta$ ranging from constant velocity $(\beta \rightarrow 0)$ to $\beta=4.5$. For high values of $\beta$ the lines are nearly symmetric, and only slightly shifted. Due to the high optical depth, the emergent lines from the corresponding homogeneous models are far too weak to be plotted to the same scale.

The most important consequence of the wind fractioning is the drastic reduction of the wind opacity. Therefore, emergent $\mathrm{X}$-ray line fluxes can be much higher than from homogeneous winds of the same mass-loss rate. The gain factor, i.e. the integrated emergent line flux ratio between a fragmented wind and the corresponding homogeneous wind, $\mathcal{R}=\log F_{\mathrm{frg}}^{*} / F_{\mathrm{h}}^{*}$, is shown in Fig. 17. The fluxes are integrated over the whole line profile. The figure displays the dependence of $\mathcal{R}$ on the radial number of fragments, $\left\langle N_{r}\right\rangle$. The different series of models have different optical depths $\tau_{*}$ (labels). The plot demonstrates that fragmentation can increase the emergent line flux by orders of magnitude. The effect is extremely pronounced for very thick winds, i.e. high $\tau_{*}$. Radial fragmentation into very many shells reduces the gain factor.

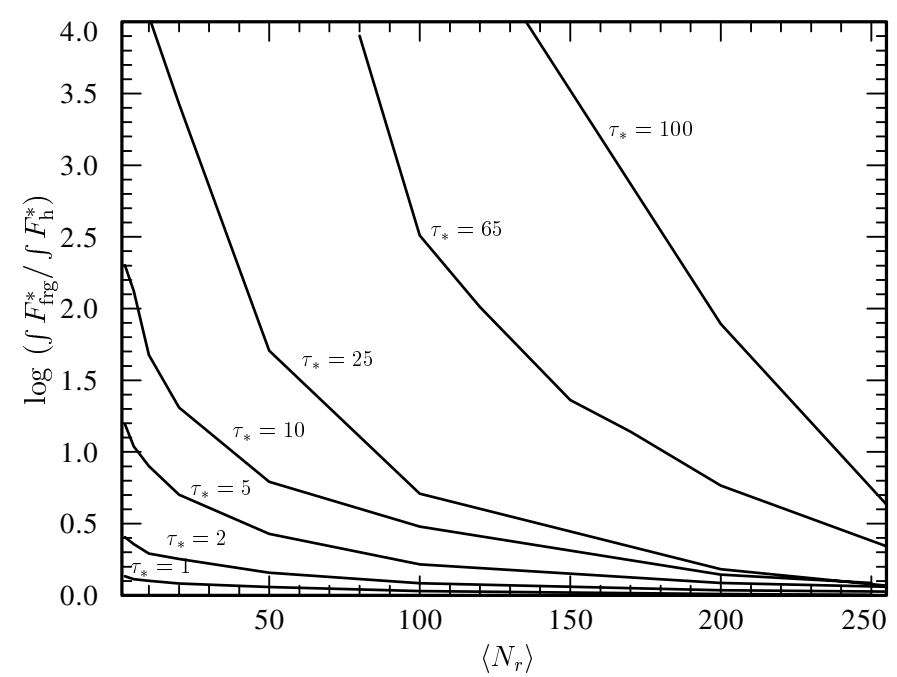

Fig. 17. The gain in emergent line flux by wind fragmentation. The ratio of the integrated line flux from fragmented and homogeneous wind models, $\mathcal{R}=F_{\mathrm{frg}}^{*} / F_{\mathrm{h}}^{*}$, is shown on a logarithmic scale. The curves show model series with different optical depths $\tau_{*}$ (labels). Along each series the number of shells in radial direction, $\left\langle N_{r}\right\rangle$, is varied. The gain factor $\mathcal{R}$ can be huge for very thick winds, i.e. high $\tau_{*}$, but is reduced with increasing number of radial fragments, $\left\langle N_{r}\right\rangle$. All models are for a velocity-law exponent $\beta=0.8$.

\section{Discussion and conclusions}

A main motivation for our fragmented wind model is to explain the observed X-ray line profiles from $\mathrm{O}$ stars, which cannot be reproduced so far. We may check now whether we came closer to this goal. Figure 18 shows the profile of the Nex line from $\zeta$ Orionis, as observed with the Chandra satellite (histogram). The smooth line is a tentative comparison with one of our calculations. From the known mass-loss rate of the star, the continuum at the frequency of this line may be optically thick (Waldron et al. 2001). Therefore we select a suitable profile (with $\beta=2$ ) from the set in Fig. 16 with $\tau_{*}=10$. The dimensionless frequency is converted into wavelength according to the terminal wind velocity of $\zeta$ Orionis $\left(2100 \mathrm{~km} \mathrm{~s}^{-1}\right)$, and the profile is convolved with a Gaussian of $0.023 \AA F W H M$ to account for instrumental broadening. The good agreement just demonstrates that our fragmented wind model can reproduce emergent line profiles of the observed shape, even if $\tau_{*}$ is large. For a systematic fit, all available line profiles should be considered in order to adjust the parameters of the model consistently. This will be subject of a forthcoming paper.

There are two aspects in the problem of fitting the observed line profiles. One is to reproduce the line shape, another is to provide for the observed line flux. Ignace \& Gayley (2002) considered profile shapes for optically thick emission lines. They showed that these lines are blueshifted, and slightly narrower than optically thin lines. The lines have a universal shape which is nearly symmetric and insensitive to the level of continuous absorption. Nevertheless, the flux in the line scales inversely with optical depth of the cool wind, and therefore the problem of missing opacity is still unresolved in their approach. The 


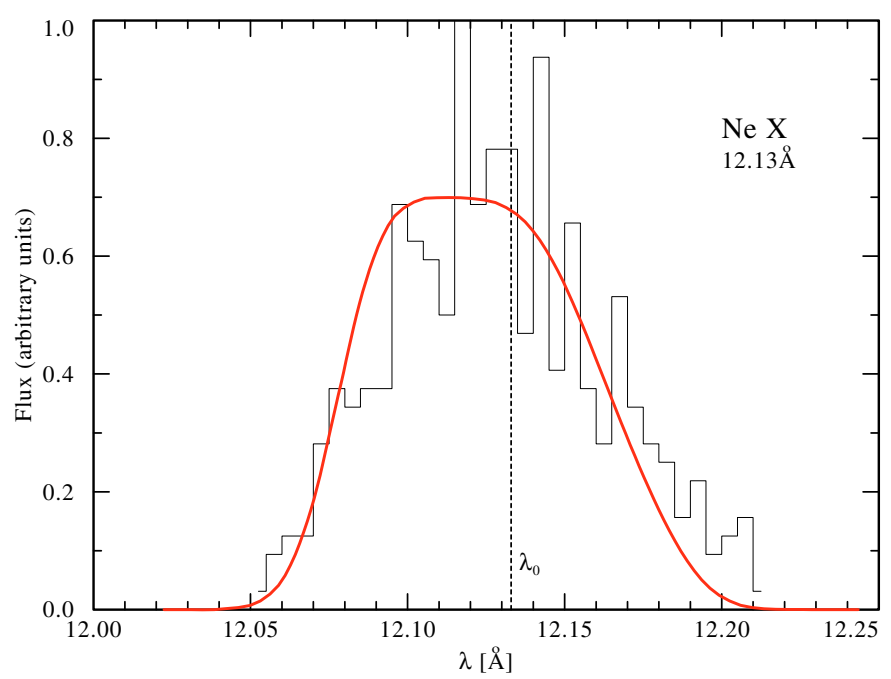

Fig. 18. Profile of the Nex line from $\zeta$ Orionis, as observed with the Chandra satellite (histogram). The smooth line is a tentative comparison with one of our calculations, taking the profile for $\tau_{*}=10$ and $\beta=2$ from Fig. 16. The theoretical profile is scaled to the terminal wind velocity of $\zeta$ Orionis $\left(2100 \mathrm{~km} \mathrm{~s}^{-1}\right)$ and convolved with the instrumental profile, a Gaussian of $0.023 \AA$ AWHM. The good fit demonstrates that our fragmented wind model can reproduce emergent line profiles of the observed shape.

same problem is highlighted in Kramer et al. (2003), which upto-day is the most consistent attempt to fit the observed lines. Our study shows that incorporation of wind fragmentation can resolve the controversy.

A burning question is, why stars with existing highresolution X-ray spectra show qualitatively different profile shapes, as listed in the introduction. At present we suggest that only $\zeta$ Pup is a safe prototype for an isolated strong stellar wind. Indeed, speckle interferometry led to the discovery that $\theta^{1}$ Ori $\mathrm{C}$ and $\zeta$ Ori A, are binary systems (Hummel et al. 2000; Weigelt et al. 1999), and $\delta$ Ori A is a well-known multiple system (see e.g., Miller et al. 2002). Moreover, $\theta^{1}$ Ori $C$ has a strong magnetic field in excess of $1 \mathrm{kG}$ (Donati et al. 2002). The physical processes leading to the emission of X-rays can differ between objects. Nevertheless, we claim that the effects of wind fragmentation ought to be included in the modeling of emerging line profiles independent of the particular emission mechanism.

The scenario chosen as a framework of the model presented here is that of Feldmeier et al. (1997), where X-ray are produced in the collision of fast cloudlets with dense shells. The prominent feature of this hydrodynamic simulation is that the $\mathrm{X}$-ray emitting plasma is always located at the starward face of the cool absorbing fragments. It was already shown in Paper I, and is confirmed here, that this configuration leads to strong depletion of the central part of the line, an effect which is certainly not observed.

The following conclusions can be drawn from our numerical modeling of the X-ray line emission from an inhomogeneous, fragmented stellar wind:

1. Fragmentation drastically reduces the effective opacity of the wind. Therefore X-rays produced deep inside the wind can effectively escape, which would be totally absorbed in a homogeneous wind of the same mass-loss rate.

2. Absorption in a fragmented wind becomes effectively independent of the mass absorption coefficient and therefore of the wavelength, if the fragments are optically thick.

3. The line profiles from the fragmented wind model exhibit a variety of shapes, ranging from broad, blueshifted and flat-topped to narrow and nearly symmetric, and are thus of promising similarity to observations.

4. The possibility of a flat-topped, i.e. almost symmetric blue part of the line profile is due to the model assumption that the fragments are flat, have small thickness, and are aligned perpendicular to the radial flow direction.

5. The effect of fragmentation is significant when the individual fragments are optically thick. Therefore the effect depends on the average number of fragments per radial direction. This parameter can be empirically restricted by a detailed line fit and has interesting implications for the theoretical understanding of the wind hydrodynamics.

6. The lateral size of the wind fragments has no influence on the emergent lines.

7. If the fragments are strictly confined to a pattern of radial cones while moving outwards, which ensures strict mass conservation in each radial direction, the resulting line profiles differ only little from the random fragment model where the absorbing shell fragments are randomly distributed in both radial and angular coordinates.

8. The numerical modeling featuring stochastically arranged emitting parcels of gas and absorbing fragments confirms the results of Paper I, where analytical formulae have been derived from a statistical treatment.

Acknowledgements. The authors are grateful to the referee John Castor, for his recommendations on future work. L.M.O. acknowledges support from a Deutsche Forschungsgemeinschaft grant (Fe 573/1-1).

\section{References}

Baade, D., \& Lucy, L. B. 1987, A\&A, 178, 213

Carlberg, R. G. 1980, ApJ, 241, 1131

Castor, J. I., Abbott, D. C., \& Klein, R. I. 1975, ApJ, 195, 157

Cassinelli, J. P., \& Swank, J. H. 1983, ApJ, 271, 681

Cassinelli, J. P., Miller, N. A., Waldron, W. L., et al. 2001, ApJ, 554, L55

Donati, J.-F., Babel, J., Harries, T. J., et al. 2002, MNRAS, 333, 55

Eversberg, T., Lepine, S., \& Moffat, A. F. J. 1998, ApJ, 494, 799

Feldmeier, A. 1995, A\&A, 299, 523

Feldmeier, A., Puls, J., \& Pauldrach, A. W. A. 1997, A\&A, 322, 878

Feldmeier, A., Oskinova, L., \& Hamann, W.-R. 2003, A\&A, 403, 217 (Paper I)

Hamann, W.-R., \& Koesterke, L. 1998, A\&A, 335, 1003

Harnden, F. R., Branduardi, G., Elvis, M., et al. 1979, ApJ, 234, 51

Hummel, C. A., White, N. M., Elias, N. M., et al. 2000, ApJ, 540, 91

Ignace, R. 2001, ApJ, 549, L119

Ignace, R., \& Gayley, K. G. 2002, ApJ, 568, 954

Kahn, S. M., Leutenegger, M. A., Cottam, J., et al. 2001, A\&A, 365, L312 
Kramer, R. H., Cohen, D. H., \& Owocki, S. P. 2003, ApJ, 592, 532

Lucy, L. B. 1982, ApJ, 255, 286

Lucy, L. B., \& Solomon, P. M. 1970, ApJ, 159, 879

Lucy, L. B., \& White, R. L. 1980, ApJ, 241, 300

MacFarlane, J. J., Cassinelli, J. P., Welsh, B. Y., et al. 1991, ApJ, 380, 564

Mihalas, D. 1978, Stellar Atmospheres, 2nd ed. (San Francisco: Freeman)

Miller, N. A., Cassinelli, J. P., Waldron, W. L., et al. 2002, ApJ, 577, 951

Morton, D. C. 1967, ApJ, 150, 535

Owocki, S. P., Castor, J. I., \& Rybicki, G. B. 1988, ApJ, 335, 914

Owocki, S. P., \& Rybicki, G. B. 1984, ApJ, 284, 337
Pauldrach, A., Puls, J., \& Kudritzki, R. P. 1986, A\&A, 164, 86

Press, W. H., Teukolsky, S. A., Vetterling, W. T., et al. 1992, Numerical recipes in FORTRAN (Cambridge University Press)

Puls, J., Repolust, T., Hoffmann, T. L., et al. 2003, in Massive star odyssey: from main sequence to supernova, ed. K. A. van der Hucht, A. Herrero, \& C. Esteban (ASP), IAU Symp., 212, 61

Schulz, N. S., Canizares, C. R., Huenemoerder, D., \& Lee, J. C. 2000, ApJ, 545, L135

Seward, F. D., Forman, W. R., Giacconi, R., et al. 1979, ApJ, 234, 55

Waldron, W. L., \& Cassinelli, J. P. 2001, ApJ, 548, L45

Weigelt, G., Balega, Y., Preibisch, Th., et al. 1999, A\&A, 347, 15 